\title{
ARTICLE
}

Received 4 May 2016 | Accepted 17 Nov 2016 | Published 10 Jan 2017

DOI: 10.1038/ncomms13991

OPEN

\section{Neuronal sFlt1 and Vegfaa determine venous sprouting and spinal cord vascularization}

\author{
Raphael Wild ${ }^{1,2}$, Alina Klems ${ }^{1,2}$, Masanari Takamiya2 ${ }^{2}$ Yuya Hayashi ${ }^{2,3}$, Uwe Strähle2, Koji Ando4, \\ Naoki Mochizuki ${ }^{4}$, Andreas van Impel ${ }^{5,6}$, Stefan Schulte-Merker ${ }^{5,6}$, Janna Krueger ${ }^{7}$, Laetitia Preau ${ }^{1}$ \\ \& Ferdinand le Noble ${ }^{1,2}$
}

Formation of organ-specific vasculatures requires cross-talk between developing tissue and specialized endothelial cells. Here we show how developing zebrafish spinal cord neurons coordinate vessel growth through balancing of neuron-derived Vegfaa, with neuronal sFlt1 restricting Vegfaa-Kdrl mediated angiogenesis at the neurovascular interface. Neuron-specific loss of flt 1 or increased neuronal vegfaa expression promotes angiogenesis and peri-neural tube vascular network formation. Combining loss of neuronal flt7 with gain of vegfaa promotes sprout invasion into the neural tube. On loss of neuronal flt1, ectopic sprouts emanate from veins involving special angiogenic cell behaviours including nuclear positioning and a molecular signature distinct from primary arterial or secondary venous sprouting. Manipulation of arteriovenous identity or Notch signalling established that ectopic sprouting in flt1 mutants requires venous endothelium. Conceptually, our data suggest that spinal cord vascularization proceeds from veins involving two-tiered regulation of neuronal sFlt1 and Vegfaa via a novel sprouting mode.

\footnotetext{
${ }^{1}$ Department of Cell and Developmental Biology, Institute of Zoology (ZOO) Karlsruhe Institute of Technology (KIT), Fritz-Haber-Weg 4, 76131 Karlsruhe, Germany. ${ }^{2}$ Institute for Toxicology and Genetics (ITG), Karlsruhe Institute of Technology (KIT), PO Box 3640, 76021 Karlsruhe, Germany. ${ }^{3}$ Department of Molecular Biology and Genetics, Aarhus University, Gustav Wieds Vej 10, 8000 Aarhus C, Denmark. ${ }^{4}$ Department of Cell Biology, National Cerebral and Cardiovascular Research Institute, 5-7-1 Fujisirodai, Suita, Osaka 565-8565, Japan. ${ }^{5}$ Institute for Cardiovascular Organogenesis and Regeneration, Faculty of Medicine, University of Münster, Mendelstr. 7, 48149 Münster, Germany. ${ }^{6}$ Cells-in-Motion Cluster of Excellence, (EXC 1003-CiM), University of Münster, Waldeyerstraße 15, 48149 Münster, Germany. ${ }^{7}$ Department of Translational Oncology, Biological Sciences Platform, Sunnybrook Research Institute, 2075 Bayview Ave., M4N 3M5 Toronto, Canada. Correspondence and requests for materials should be addressed to F.I.N. (email: ferdinand.noble@kit.edu).
} 
T he vascular network closely associates with the neuronal network throughout embryonic development, in adulthood and during tissue regeneration ${ }^{1-3}$. Close association of vessels and nerves allows reciprocal cross-talk involving diffusible molecules, which is important for physiological functions in both domains ${ }^{4,5}$. Arteries secrete factors that attract sympathetic axons, and adrenergic innervation of arteries allows the autonomic nervous system to control arterial tone and tissue perfusion ${ }^{5}$. The nervous system, on the other hand, requires a specialized network of blood vessels for its development and survival. Metabolically active nerves rely on blood vessels to provide oxygen necessary for sustaining neuronal activity $^{6}$, and disturbances herein result in neuronal dysfunction ${ }^{1,7}$

How nerves attract blood vessels is debated, but several studies addressing vascularization of the mouse and chicken embryonic nervous system suggest that the angiogenic cytokine VEGF-A is involved ${ }^{8-10}$. In the mouse peripheral nervous system axons of sensory nerves innervating the embryonic skin trigger arteriogenesis involving VEGF-ANeuropilin-1 (NRP1) dependent signalling ${ }^{11,12}$. While these studies provide evidence for the physical proximity and cooperative patterning of the developing nerves and vasculature, relatively little is known about mechanisms controlling VEGF-A dosage at the neurovascular interface. This is of great importance considering that blood vessels are very sensitive to changes in VEGF-A protein dosage and even moderate deviations from its exquisitely controlled physiological levels result in dramatic perturbations of vascular development ${ }^{13,14}$. VEGF-A levels must therefore be well titrated, and several strategies have evolved to achieve this.

Mouse retinal neurons for example can reduce extracellular VEGF-A protein via selective endocytosis of VEGF-A-VEGF receptor-2 (KDR/FLK) complexes. Inactivation of this uptake causes non-productive angiogenesis ${ }^{15}$. In the vascular system, spatio-temporal control of VEGF-A protein dosage is thought to be achieved by soluble VEGF receptor-1 (sFLT1), an alternatively spliced, secreted isoform of the cell-surface receptor membranebound FLT1 (mFLT1) $^{16,17}$. Soluble FLT1 binds VEGF-A with substantially higher affinity than KDR, thereby reducing VEGF-A bioavailability and attenuating KDR signalling ${ }^{17}$. While originally discovered as a vascular-specific receptor, evidence is emerging showing neuronal FLT1 expression ${ }^{18}$. To what extent endogenous neuronal Flt1 has a physiological role in titrating neuronal VEGF levels controlling angiogenesis at the neurovascular interface independent of vascular Flt1 remains to be determined.

Angiogenesis involves complex and dynamic changes in endothelial cell behaviour ${ }^{19}$. In the zebrafish embryo these events can be studied in detail at the single cell level in vivo through the use of vascular-specific reporter lines ${ }^{20,21}$. The stereotyped patterning of arteries and veins in the trunk of the zebrafish embryo prior to $48 \mathrm{hpf}$ is mediated by cues derived from developing somites and the hypochord, controlling angiogenic sprout differentiation and guidance ${ }^{22,23}$. Sprouting of intersegmental arterioles (aISV) requires Vegfaa-Kdrl signalling, as loss of either $k d r l$ or vegfaa completely abolishes ISV sprouting from the dorsal aorta (DA) ${ }^{24}$. Primary sprouting also involves a component regulated by Notch, as loss of Notch increases the endothelial propensity to occupy the tip cell position in this vessel, whereas gain of Notch restricts aISV development ${ }^{25}$. Secondary vein sprouting requires Vegfc-Flt4 signalling, as loss of either ligand or receptor blocks venous growth ${ }^{26,27}$. Developing somites are regarded as the main source for Vegfaa, while the hypochord provides Vegfc during early development ${ }^{22,23}$.
In this study we show that developing spinal cord neurons located in the trunk of the zebrafish embryo produce Vegfaa and sFlt1 affecting the angiogenic behaviour of intersegmental vessels at the neurovascular interface. We find that during early development neuronal sFlt1 restricts angiogenesis around the spinal cord. We demonstrate that on genetic ablation of neuronal sFlt1 this brake is relieved resulting in the formation of a vascular network supplying the spinal cord in a Vegfaa-Kdrl dependent manner. Using inducible neuron-specific vegfaa gain-of-function approaches and analysis of several mutants with vegfaa gain-of-function scenarios, we furthermore show that the neuronal Vegfaa dosage determines the extent of the neovasculature supplying the spinal cord, as well as sprout invasion into the spinal cord. Interestingly, loss of flt 1 or augmenting neuronal vegfaa promotes sprouting from intersegmental veins involving distinctive angiogenic cell behaviours including nuclear positioning and a molecular signature not observed in primary arterial or secondary venous sprouting. Cell transplantation experiments confirm the role of neuronal $f t t 1$ in venous sprouting and furthermore show that vascular $f t t 1$ is dispensable herein. Taken together, our data suggest that spinal cord vascularization proceeds from veins and is coordinated by two-tiered regulation of neuronal sFlt1 and Vegfaa determining the onset and the extent of the vascular network that supplies the spinal cord via a novel sprouting mode.

\section{Results}

Spinal cord neurons express sflt1, mflt1 and vegf ligands. Analysis of TgBAC(flt1:YFP $)^{h u 4624} ; \mathrm{Tg}(\mathrm{kdrl}: \text { hsa.HRAS-mCherry })^{s 916}$ transgenic embryos showed flt1 expression in the aorta, arterial intersegmental vessels (aISVs), dorsal part of venous intersegmental vessels (vISVs) and spinal cord neurons located in the neural tube (Fig. $1 \mathrm{a}, \mathrm{b}, \mathrm{d}-\mathrm{g})^{18}$. Spinal cord neurons were in close proximity to blood vessels (Fig. 1c-e) and 3D-rendering of confocal z-stacks obtained from $\mathrm{Tg}(\mathrm{kdrl}: \mathrm{EGFP})^{\text {s843 }} ; \mathrm{Tg}(\mathrm{Xla} \text {.Tubb:DsRed })^{z f 148}$ double transgenic embryos showed the dorsal aspect of ISVs 'indenting' the neural tube indicative of close contact (Fig. 1c; Supplementary Movie 1). Optical sections confirmed close contact between the outer neuronal layers of the neural tube and the dorsal part of ISVs, as well as the dorsal longitudinal anastomotic vessel (DLAV) (Fig. 1d-g). Such anatomical juxtapositioning of trunk vessels and neurons may provide a template for molecular cross-talk (Fig. 1d,e; pink box).

TaqMan analysis using FAC-sorted neuronal cells from two different neuronal reporter lines (Supplementary Fig. 1a-k) showed expression of $m f l t 1, s f l t 1, k d r l, k d r$, flt 4 and the ligands vegfaa, vegfab, and plgf (Supplementary Fig. 1b,e $)^{24}$. Flt1 was expressed in a comparable range as neuronal guidance molecules (Supplementary Fig. 1c,f). Real-time qPCR analysis for vegfa a and vegfab in the trunk of developing zebrafish embryos confirmed expression of both isoforms (Supplementary Fig. 11,m).

Loss of flt1 induces ectopic vascular networks. In zebrafish flt 1 consists of 34 exons encoding membrane-bound $m f l t 1$ and soluble $s f t 1$, which is formed by alternative splicing at the exon 10 -intron 10 boundary (Supplementary Fig. 2a) $)^{18}$. To obtain loss of both mflt1 and sflt1 (flt1 full mutants) we targeted flt1-exon 3 using a CRISPR/Cas approach (Supplementary Fig. 2a-d) and analysed in detail the vascular phenotypes of three mutant alleles, $f t t 1^{k a 601}(-1 \mathrm{nt}), \mathrm{flt}^{\mathrm{ka602}}(-5 \mathrm{nt})$ and $f_{t t}{ }^{k a 603}(+5 \mathrm{nt})$ (Fig. 2a-f; Supplementary Fig. 2a-d). To obtain mflt1-specific mutants we targeted exon 11b, the alternative exon essential for $m f l t 1$ transcription (Fig. 2g; Supplementary Fig. 2a,e $)^{18}$. Both the $f l t 1^{k a 601}$ and $f l t 1^{k a 605}$ mutant showed no signs of non-sense mediated decay (Supplementary Fig. 3a,b). 

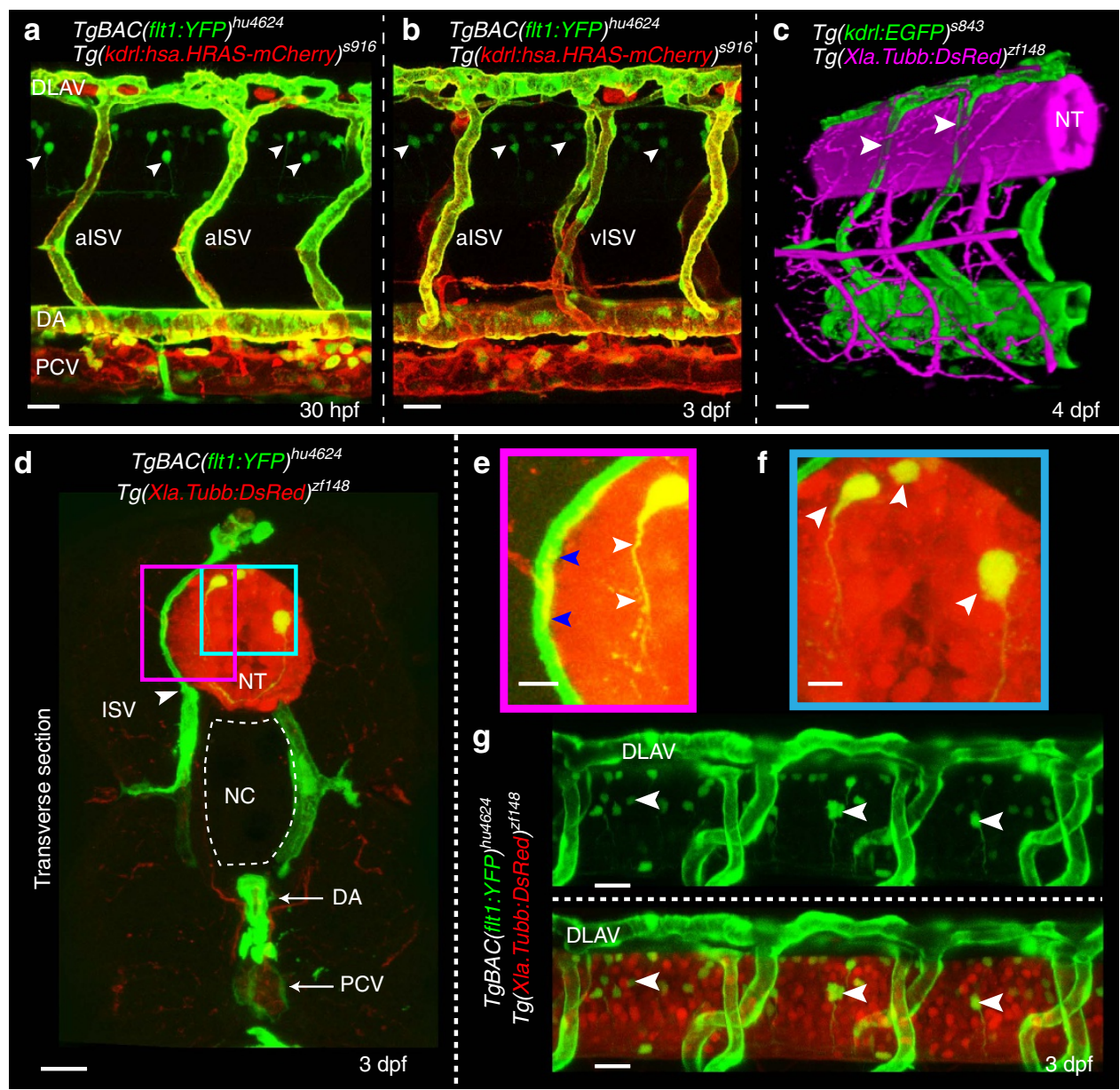

Figure 1 | Expression of Vegf receptors and ligands at the neurovascular interface. (a,b) Double transgenic embryos TgBAC(flt7:YFP) ${ }^{h u 4624}$;

$\mathrm{Tg}$ (kdrl:hsa.HRAS-mcherry) ${ }^{916}$ at $30 \mathrm{hpf}$ and $3 \mathrm{dpf}$ shows flt1 expression (green) in dorsal aorta, arterial ISV and dorsal aspect of venous ISV (3 dpf) and neurons (arrowheads). (c) 3D-rendered view of vessels (green) and nerves (purple) in Tg(kdrl:EGFP)s843;Tg(Xla.Tubb:DsRed) ${ }^{\text {ff148 }}$ embryos highlighting dorsal aspect of ISVs (arrowheads) in close contact to the neural tube (NT). (d) Transverse section of the trunk of TgBAC(flt1:YFP) hu4624;

$\mathrm{Tg}$ (Xla.Tubb:DsRed) ${ }^{\text {zf148 }}$ embryos shows that ISVs (green, arrowhead) and neural tube (NT, red) are in close contact. Dorsal is up. (e) Magnified view of purple-boxed area in (d), showing direct contact of vessels with nerves at the neurovascular interface (blue arrowheads) and flt1 expressing neurons with long axonal extensions in the neural tube (white arrowheads). (f) Magnified view of blue-boxed area in (d) showing flt1 expressing neurons (arrowheads) and their axons inside neural tube (red). (g) Lateral view of TgBAC(flt1:YFP) hu4624; Tg(Xla.Tubb:DsRed) ${ }^{\text {zf148 }}$ at the level of the neural tube showing flt1 expressing neurons (arrowheads) in neural tube. DA, dorsal aorta; dpf, days post fertilization; DLAV, dorsal longitudinal anastomotic vessel; hpf, hours post fertilization; ISV, intersegmental vessel; NC, notochord; NT, neural tube; PCV, posterior cardinal vein. Scale bar, $30 \mu m$ in $\mathbf{a}-\mathbf{d}, \mathbf{g} ; 10 \mu \mathrm{m}$ in $\mathbf{e , f}$.

Zebrafish homozygous for the $f t t 1-1$ nt allele $\left(f l t 1^{k a 601}\right)$ displayed severe hyper-branching of the trunk vasculature at $3-4 \mathrm{dpf}$ (Fig. 2a,b). Supernumerous amounts of branches developed in the dorsal aspect of the trunk at the level of the neural tube (Fig. 2b,c). Comparable observations were made in embryos homozygous for the $f t t 1-5 \mathrm{nt}$ allele $\left(f l t 1^{k a 602}\right)$ and the flt $1+5 \mathrm{nt}$ allele $\left(\mathrm{flt}_{1} \mathrm{ka603}^{\mathrm{s}}\right.$ ) (Fig. 2d,e,f). Analysis of four $m f l t 1$ mutant alleles ( $f l t 1^{\text {ka605-608 }}$, Supplementary Fig. 2e) did not reveal any obvious vascular malformations or alterations in vascular branching morphogenesis (Fig. $2 \mathrm{~g}$ ). These observations are compatible with absence of angiogenic defects in mouse Flt $1^{T K-} f_{-}$embryos lacking mFlt1 signalling ${ }^{28,29}$. The vascular phenotype observed in the $f l t 1^{k a 601}$ mutants thus most likely involved soluble Flt1.

Since the vascular phenotypes of the $f t 1^{k a 601}, f l t 1^{k a 602}$ and $f l t 1^{k a 603}$ mutant alleles ( $f t t 1$ full mutants) were indistinguishable, we focused on analysing $f t 1^{k a 601}$ embryos (Fig. 2p-s). Flt $1^{\text {ka601 }}$ mutants showed normal arterial-venous remodelling (Fig. 2b,p-s) and adequate perfusion of both aISVs and vISVs.
No significant changes in heart frequency were noted (Supplementary Fig. 4a). The vascular phenotype of $f t 1^{k a 601}$ mutants emerged around day 2.5 (Fig. 2q,r) with sprouts emanating exclusively from the dorsal aspect of the venous ISVs at the level of the neural tube (Supplementary Movie 2); ectopic arterial ISV sprouting was not observed (Fig. 2r). In flt $1^{\text {ka601/+ }}$ heterozygotes (Fig. 2l-o) ectopic sprouting was rarely observed (Fig. 2n,o; Supplementary Movie 3). In wild-type (WT) embryos such endothelial cell behaviours were not observed (Fig. 2h-k, Supplementary Movie 4).

We furthermore examined whether flt1 targeting morpholino could recapitulate the $f l t 1^{k a 601}$ mutant phenotype (Supplementary Fig. 4b-h). We evaluated two dosages of a published flt 1 ATG targeting morpholino (MO) and found that $1 \mathrm{ng} f t t$ MO induced hyper-branching in WT at levels comparable to $f t 1^{k a 601}$ (Supplementary Fig. 4g,h) ${ }^{18,30}$. Injection of $1 \mathrm{ng}$ MO into $f t 1^{k a 601}$ mutant background did not induce additional sprouting defects (Supplementary Fig. 4d), suggesting that the 1ng dosage targets flt 1 specifically. In contrast, $3 \mathrm{ng} \mathrm{MO}$ introduced 

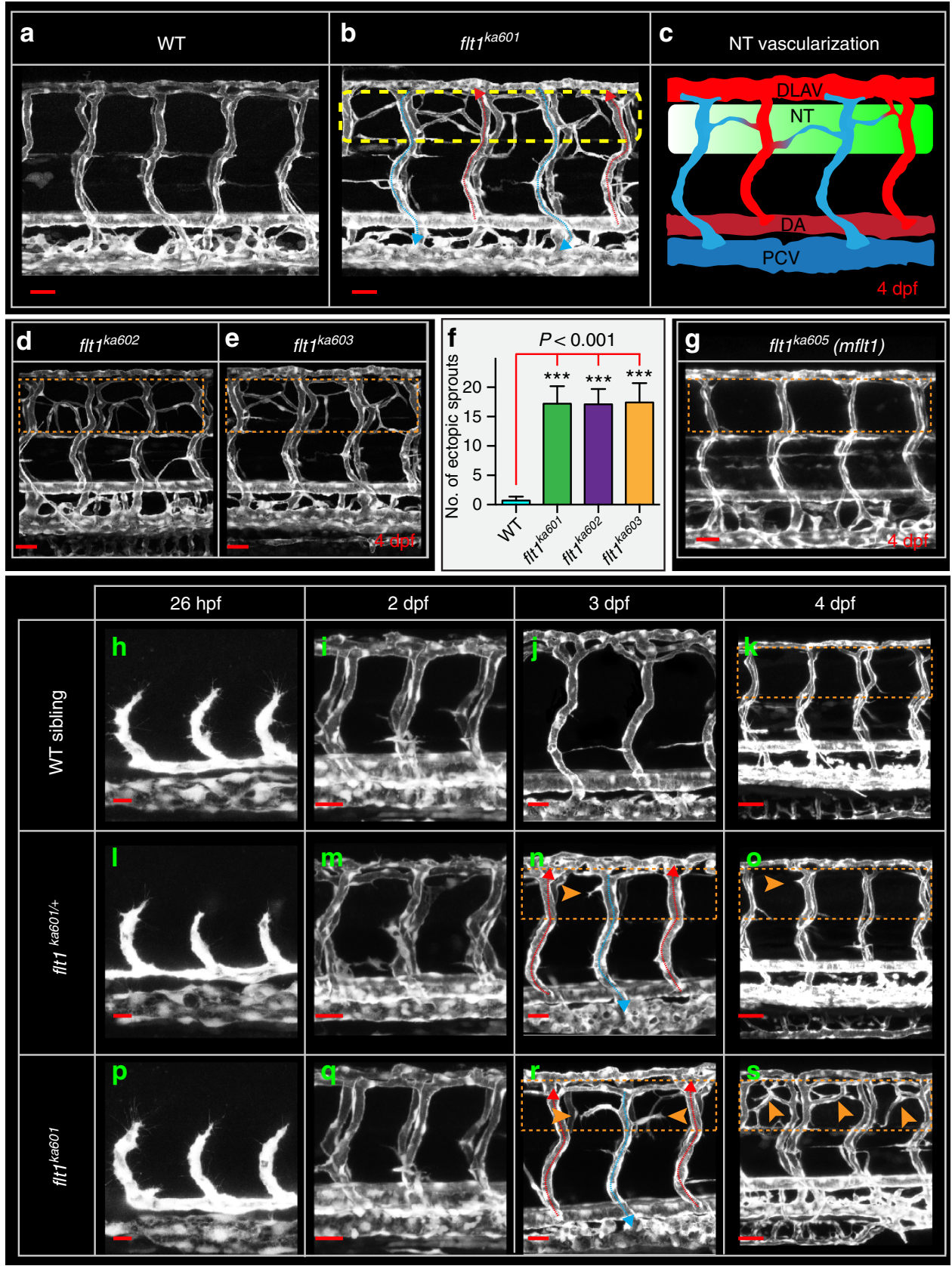

Figure 2 | FIt1 mutants develop hyper-branched vascular networks at the level of the neural tube. (a) Trunk vasculature in 4 dpf WT sibling, (b) trunk vasculature in $4 \mathrm{dpf} f(t)^{k a 601}$ mutant, in $T g(k d r l: E G F P)^{s 843}$ background. Perfused alSVs with red arrow, veins with blue arrow. Note the extensive amount of hyper-branching (dotted box) at the level of the neural tube. (c) Schematic representation of hyper-branching phenotype along the neural tube; ectopic vessels make anastomosis between vISV (blue) with alSVs (red). (d) Hyper-branching (dotted box) is also observed in $f(t)^{k a 602}$ and (e) $f(t)^{k a 603}$ mutants. (f) Quantification of hyper-branching for indicated mutant alleles. Mean \pm s.e.m., $n=10$ per group, ANOVA. (g) Membrane-bound flt7 mutant (flt ${ }^{k a 605}$ ) without vascular phenotype (compare dotted box in $\mathbf{g}$, with control in a). (h-k) Trunk vascular network in WT embryos at indicated time points. (I-o) Trunk vasculature in flt $7^{k a 601 /+}$ embryos at indicated time points. (p-s) Trunk vasculature in flt $7^{k a 601}$ embryos at indicated time points. Arrowheads indicate ectopic branches. DA, dorsal aorta; PCV, posterior cardinal vein; DLAV, dorsal longitudinal anastomotic vessel; NT, neural tube; hpf, hours post fertilization; dpf, days post fertilization. Scale bar, $50 \mu \mathrm{m}$ in $\mathbf{a}, \mathbf{b}, \mathbf{d}, \mathbf{e}, \mathbf{g}, \mathbf{i}, \mathbf{m}, \mathbf{q}, \mathbf{k}, \mathbf{0}, \mathbf{s} ; 25 \mu \mathrm{m}$ in $\mathbf{h}, \mathbf{l}, \mathbf{p}, \mathbf{j}, \mathbf{n}, \mathbf{r}$.

additional branches at $2 \mathrm{dpf}$ that were not observed in the $f l t 1^{k a 601}$ mutant at this stage (Supplementary Fig. 4e). Since we did not observe maternal contribution of flt 1 these observations suggest that $3 \mathrm{ng} \mathrm{MO}$ introduced non-specific effects ${ }^{31}$.

Sprouts in flt $^{\text {ka601 }}$ display distinctive cell behaviours. Compatible with ectopic sprouting we identified hyperactive endothelial cells extending filopodia in the dorsal aspect of vISVs of $f l t 1^{k a 601}$ mutants (Fig. 3a; Supplementary Movie 2). About 55\% of hyperactive endothelial cells investigated generated a patent sprout (Fig. 3a); in the remaining 45\%, filopodia and sprouts retracted (Fig. 3b). From the population of patent ectopic venous sprouts $95 \%$ formed an anastomosis with an aISV, whereas only $5 \%$ made a connection with a vISV (Fig. 3c). The preference for arterial anastomosis may be physiologically 

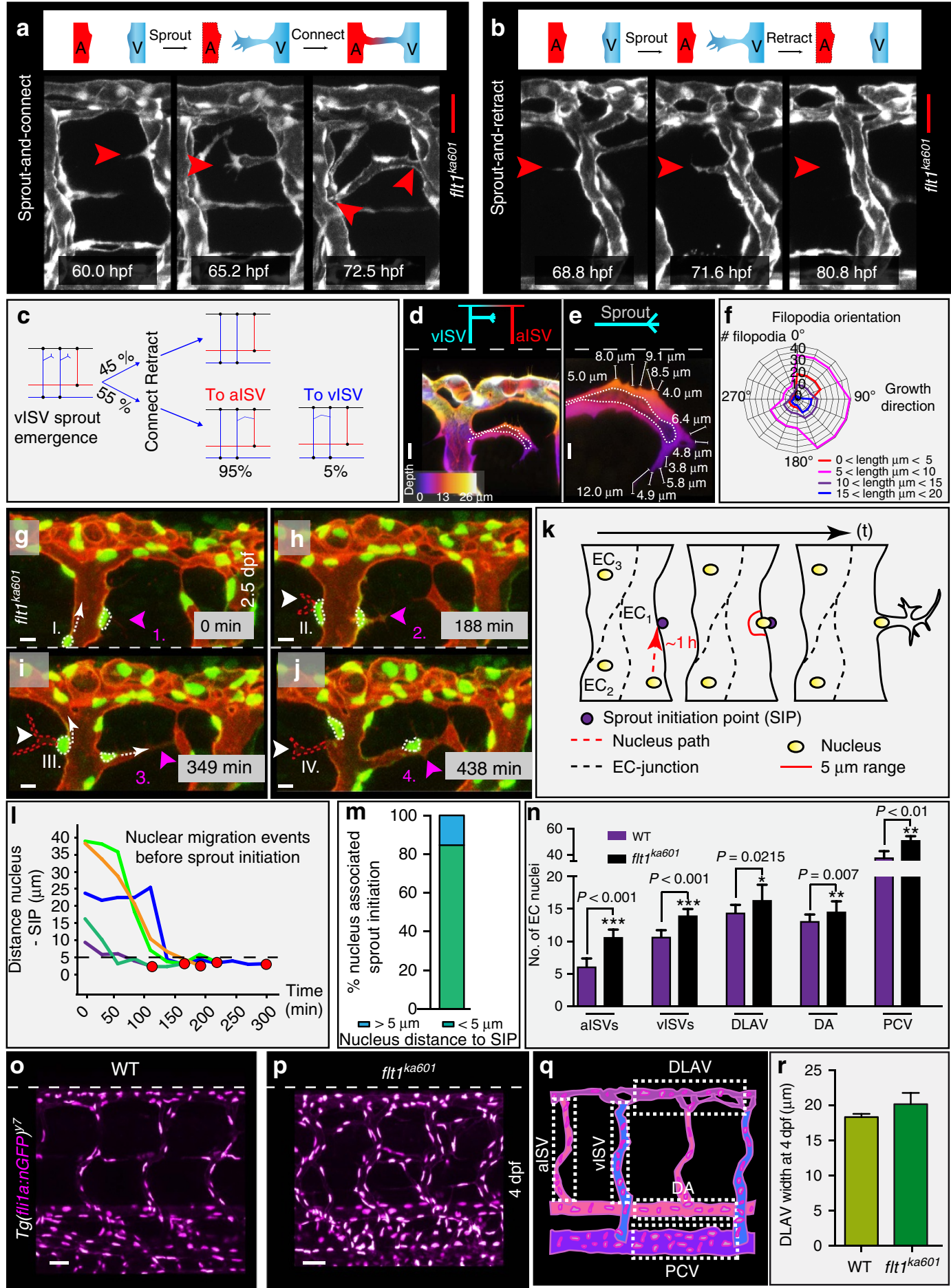

Figure 3 | Imaging and quantification of sprouting kinetics in flt $\mathbf{k a 6 0 1}^{\mathbf{a}}$ mutants. (a) Time lapse imaging of sprout initiation and anastomosis formation in $f l t \mathrm{k}^{\mathrm{k} 601}$ mutant. Sprout initiation ( $60.0 \mathrm{hpf}$ ), elongation (65.2 hpf) and connection-anastomoses ( $72.5 \mathrm{hpf}$ ) with adjacent alSV. (b) Time lapse imaging of sprout initiation and retraction in $\mathrm{flt} \mathrm{t}^{\mathrm{ka} 601}$ mutant. Endothelial cells produce filopodia ( $\left.68.8 \mathrm{hpf}\right)$, extend a sprout ( $\left.71.6 \mathrm{hpf}\right)$, which subsequently retracts (80.8 hpf). (c) Quantification of data in (a,b) showing \% of sprouts retracting (top part, 45\%) or connecting (bottom part, 55\%) to adjacent ISVs. In the latter scenario sprouts in $95 \%$ of cases connected to alSV (red) and in $5 \%$ of cases to vISV (blue). Angiogenic behaviour was analysed in time-lapse confocal movies, $n=20$ embryos. (d-f) Filopodia directionality and length in flt $7^{k a 601}$ mutants ( $n=10$ embryos, $n=920$ filopodia). (g-j) Time lapse imaging of endothelial nuclei in $\mathrm{Tg}$ (fli7a:nGFP)y7; $\mathrm{Tg}$ (kdrl:hsa.HRAS-mcherry) ${ }^{\mathrm{s} 916}$ showing association between nuclear position and sprouting initiation point (SIP). Note that sprouts arise in close proximity to the position of the nucleus. Arrowheads indicate sprouts; nuclei at indicated time points (sprout initiation with actively migrating nucleus towards SIP I, II, III, IV and nucleus already located at SIP 1,2,3,4). (k) Schematic representation of nuclear position with respect to SIP. (I,m) Quantification of observations in $\mathbf{g}-\mathbf{j}$. Red dot indicates sprout initiation time point. Note that sprouting preferentially occurs when endothelial nuclei are within less than $5 \mu \mathrm{m}$ from SIP (SIP below dotted line in (I)). $n=5(\mathbf{I})$ and $n=13(\mathbf{m})$. (n-q) Quantification of EC nuclei in alSV, vISVs, DLAV, DA and PCV of WT and $f \mid t t^{k a 601}$ embryos at $4 \mathrm{dpf}$; mean \pm s.e.m., $t$-test, $n=21$ embryos per genotype. (r) Quantification of DLAV width in WT and flt $7^{k a 601}$ mutant, $n=9$ embryos per genotype. A, artery; alSV, arterial intersegmental vessel; DA, dorsal aorta; DLAV, dorsal longitudinal anastomotic vessel; EC, endothelial cell; PCV, posterior

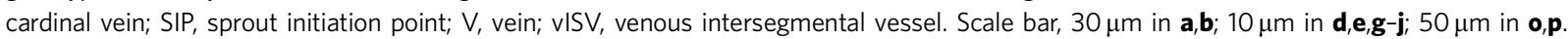


relevant as it creates a pressure gradient promoting blood flow perfusion. Sprout filopodia length ranged from 1 to $20 \mu \mathrm{m}$, and filopodia projected at an angle between 90 and $120^{\circ}$ with respect to the vISV compatible with arterial anastomosis formation (Fig. 3d-f). Current models posit that Flt1 produced in angiogenic sprouts mainly prevents back-branching of nascent sprouts $^{32}$. We find that in the absence of Flt1 sprouts retain their directionality and migrate away from the parent vessel. Within ISVs endothelial nuclei migrated at velocities of up to $1 \mu \mathrm{m} \mathrm{min}^{-1}$ (Fig. $\left.3 \mathrm{~g}-\mathrm{j}, \mathrm{l}\right)$. Careful analysis of nuclear positioning within endothelial cells revealed an association between nuclear position and sprout initiation (Fig. 3k-m, Supplementary Movie 2). Nuclei migrated actively into the direction of future sprout initiation points (SIP), and in more than $80 \%$ of the studied sprout initiations nuclear positioning was directly linked with sprout initiation (linkage was defined as nucleus-SIP distance of $<5 \mu \mathrm{m}$ at spout initiation) (Fig. 3l,m). This nuclear migration behaviour is in contrast to rearward nuclear positioning in migrating angiogenic endothelial cells in vitro ${ }^{33}$ and is not described in vivo for primary artery or secondary venous sprouting events in zebrafish. Analysis of endothelial cell numbers at $4 \mathrm{dpf}$ showed increased endothelial cell numbers in aISVs, vISVs, DLAV, DA and PCV of $f t 1^{k a 601}$ compared with WT (Fig. 3n-q); DLAV size was not statistically different (Fig. 3r). At earlier stages (17 hpf) we found no differences in endothelial cell numbers between $f l t 1^{k a 601}$ and WT (Supplementary Fig. 4i-1).

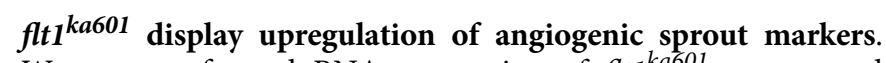
We next performed RNA sequencing of $f t 1^{k a 601}$ mutants and analysed genes implicated in sprouting angiogenesis (Supplementary Fig. 5a-c). Expression of the classical tip-stalk cell markers including notch $1 a$, notch $1 b$, dll4, nrarpa, nrapb, hey1, hey2, her6 and flt4 were not altered ${ }^{34}$ (Supplementary Fig. 5b). This result may not be surprising since ectopic venous sprouts emanated from venous ISVs, and Dll4-Notch signalling is absent in this domain ${ }^{35}$. Instead we found upregulated expression of other genes implied in sprouting cell behaviour. RNA-seq and qPCR of flt1 mutants showed significantly increased levels of apelin receptor-a (aplnra), angiopoietin-2a (angpt2a), and endothelial cell specific molecule-1 (esm1) (Supplementary Fig. 5b,c), genes previously shown to be enriched in angiogenic vessels ${ }^{36,37}$. In addition, we observed a significant upregulation of plgf, which encodes the Flt1-specific pro-angiogenic ligand Plgf, and lyve1, a gene expressed in veins and implied in lymphangiogenesis, in line with the venous expansion phenotype in $f l t 1^{\text {ka601 }}$ mutants (Supplementary Fig. $\left.5 \mathrm{c}\right)^{38}$.

Origin of endothelial cells in ectopic venous sprouts. It is established that artery-derived ECs, on arteriovenous (AV) remodelling, contribute to the dorsal aspect of vISVs (ref. 39). Besides these remodelled artery-derived cells, another source may be PCV-derived venous endothelial cells as they can migrate over long distances ${ }^{40}$. However, a specific contribution of these venous ECs in populating the dorsal aspect of vISVs has not been shown thus far. To determine whether PCV-derived venous cells can colonize the dorsal aspect of vISVs, we performed cell tracking experiments using the $T g(k d r l: n l s k i k G R)^{h s c 7}$ transgenic line (Fig. 4a-i). A small part of the PCV was photo-converted at $30 \mathrm{hpf}$ and individual venous endothelial cells were tracked in the period $30-60 \mathrm{hpf}$ by time-lapse imaging (Fig. 4a-f, Supplementary Movie 5). We observed three scenarios (Fig. 4i). In scenario (I): PCV-derived venous endothelial cells migrated into the vISV and reached the most dorsal aspect of the vISV (Fig. 4c-e). In the dorsal aspect of vISVs, PCV-derived endothelial cells were observed together with the remodelled artery-derived endothelial cells (Fig. 4f,g; artery-derived cells in green). Scenario (I), which we refer to as 'mixed' (both artery and vein-derived EC), accounted for $43.2 \%$ of cases (Fig. 4h,i). Of the mixed population $67.9 \%$ of endothelial cells were of venous origin and $32.1 \%$ of arterial origin (Fig. 4h, right panel). In scenario (II), the dorsal part of vISV only contained PCV-derived venous endothelial cells; artery-derived endothelial cells were absent. Scenario (II) accounted for $48.6 \%$ of cases (Fig. $4 \mathrm{~h}, \mathrm{i}$ ). In scenario (III) we find that the dorsal part of vISV only contained artery-derived ECs; in this scenario the dorsal aspect of vISVs was not colonized by migrating PCV-derived venous endothelial cells (Fig. 4h,i). This scenario was observed in $8.2 \%$ of cases.

The $f t 1^{\text {enh }}$ promoter marks ISV-ECs of arterial origin ${ }^{39}$. Loss of $f l t 1$ in $\mathrm{Tg}\left(\mathrm{flt} \mathrm{1}^{\text {enh }}\right.$ :Tdtomato; flt4:mCitrine) showed ectopic venous sprouts containing $f l t 1^{\text {enh }}$-expressing ECs (Fig. $4 \mathrm{k}, 1, \mathrm{n}-\mathrm{p}$ ). In the same embryo, we furthermore noted ectopic venous sprouts devoid of $f l t 1^{\text {enh }}$ expressing ECs (Fig. 4j,m,p), suggesting that these sprouts were only made of vein-derived ECs (Fig. $4 \mathrm{~m}$ ). To confirm a contribution of PCV-derived venous endothelium we performed cell tracking experiments in $T g(k d r l: n l s k i k G R)^{h s c 7}$ on loss of $f l t 1$ and indeed we found that PCV-derived venous ECs were capable of contributing to ectopic sprouting (Supplementary Fig. 6a). Interestingly, besides sprouts exclusively containing artery-derived, or venous-derived endothelium (Fig. 4m,o), we observed composite sprouts with artery and venous-derived endothelial cells juxtapositioned (Fig. 4k,n).

Vegfaa gain-of-function promotes venous sprouting. Before $48 \mathrm{hpf}$ trunk arterial sprouting is driven by Vegfaa and venous sprouting by Vegfc (refs $24,26,27$ ). Since loss of flt 1 mimics vegfaa gain-of-function, we expected changes in arterial branching in $f t 1^{k a 601}$. Rather surprisingly, we observed ectopic venous sprouting after $2.5 \mathrm{dpf}$ (Fig. 3a,b; Fig. 5a,b,e). Primary artery development was not affected in flt 1 mutants (Supplementary Fig. 6b,c,f,g), although primary arterial sprouts developed in close proximity to the neural tube (Supplementary Fig.6h-k).

Ectopic venous sprouting was conserved in several other vegfaa gain-of-function scenarios, including $v h l^{h u 2114}$ mutants and ptena ${ }^{-/-} ;$pten $b^{-/-}$double mutants (Fig. 5c-e). Von Hippel-Lindau protein (pVHL) is essential for the proteolytic degradation of Hif- $1 \alpha$, an evolutionary conserved transcription factor important for regulating vegfaa transcription $^{41,42}$. Loss of $v h l$ prevents Hif- $1 \alpha$ degradation and augments vegfaa expression ${ }^{41,42}$. Accordingly, vhh ${ }^{\text {hi2114 }}$ mutants developed ectopic sprouts emanating from vISVs but not from aISVs (Fig. 5c,e). Changes in primary aISV sprouting were not observed (Supplementary Fig. 6d,f,g).

PTEN is a tumour suppressor gene acting as a PI3K/Akt signalling attenuator and linked to the progression of many tumours involving VEGF-A (refs 43,44). In zebrafish, two orthologues of pten exist, and ptena ${ }^{-/-} ;$pten $^{-/-}$double mutant zebrafish show increased vegfaa levels ${ }^{44}$. Detailed analysis of ptena $a^{-/-} ;$pten $b^{-/-}$double mutants identified pronounced ectopic venous sprouting at the level of the neural tube (Fig. 5d,e). In pten double mutants ectopic venous sprout numbers were higher when compared with $f t 1^{k a 601}$ single mutant or $v h l^{h u 2114}$ single mutant (Fig. 5e). In addition, in a small percentage of ISVs, ptena ${ }^{-/-} ;$pten $b^{-/-}$double mutants displayed very few ectopic arterial sprouts (Fig. 5e).

Mechanistically, loss of $v h l$ and $f l t 1$ augments Vegfaa function at different levels, through increased vegfaa transcription and higher Vegfaa protein bioavailability, respectively. We reasoned that combining both mutants should increase Vegfaa 

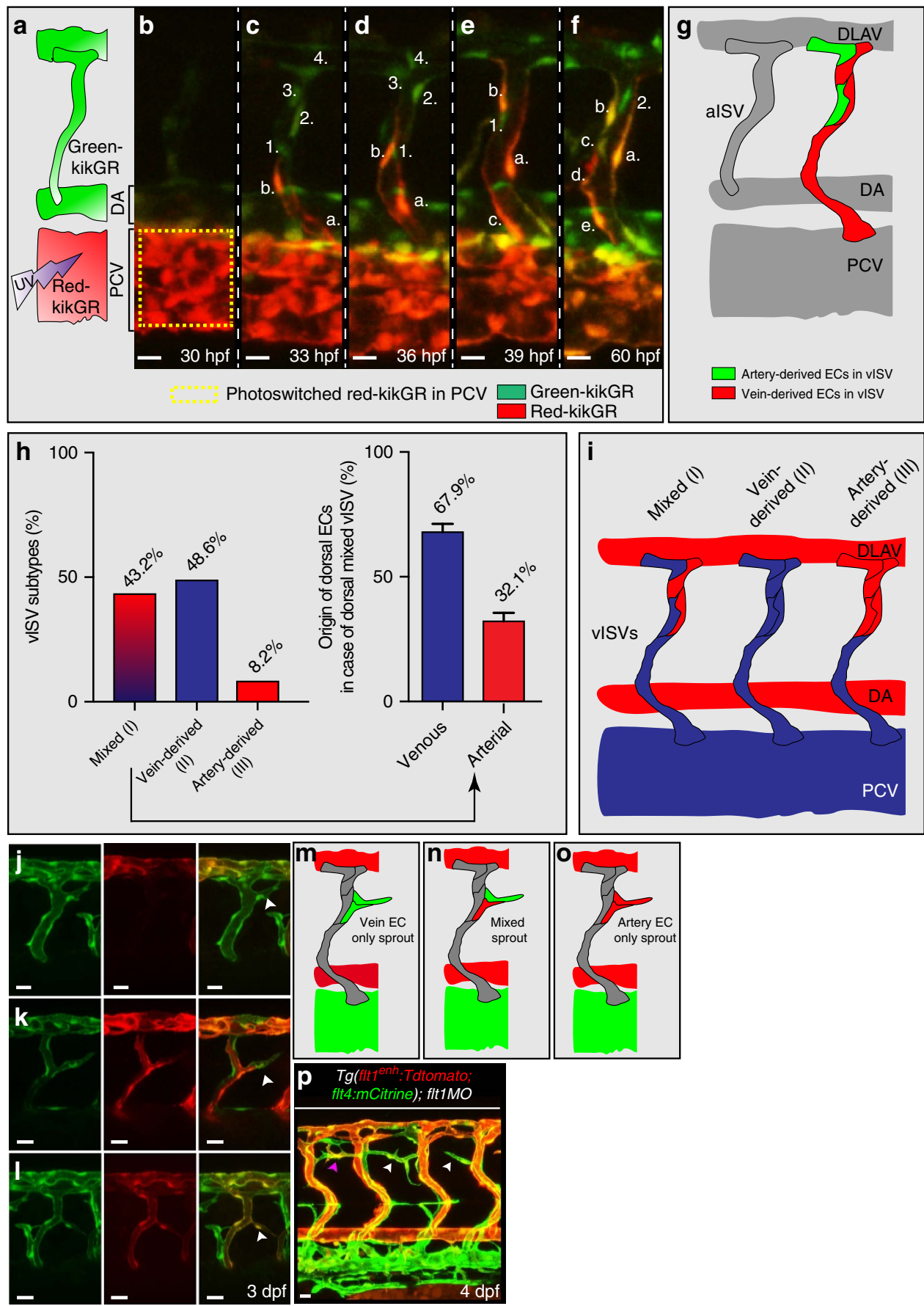

Figure 4 | Arterial and venous-derived endothelial cells populate the dorsal part of venous ISV and contribute to sprouting upon loss of flt1.

(a) Endothelial cell tracking in $T g(k d r l: n l s K i k G R)^{h s c 7}$ embryos. Photo-converted PCV-derived venous endothelial cells express red-kikGR. (b-f) Endothelial cell tracing during 30-60 hpf, showed that PCV-derived endothelium, indicated in red \& labelled a,b,c,d,e, migrated along the ISV, from ventral to dorsal up to the most dorsal part of vISVs (cell labelled b). Pre-existing arterial endothelial cells in ISV, in green and labelled 1,2,3,4, shows artery-derived ECs in the dorsal part of vISVs (cell labelled 2) adjacent of PCV-derived EC (cell labelled $\mathbf{b}, \mathbf{a}$ ). (g) Schematic representation of the scenario imaged in $\mathbf{b}-\mathbf{f}$. (h) Left panel: Identity analysis of endothelial cells in the dorsal part of vISV revealed three different scenarios: (I) mixed, both arterial and venous-derived endothelium were present, (II) only vein-derived endothelium, (III) only artery-derived endothelium. ( $n=10$ experiments \& 6ISVs/embryo). Right panel: $\%$ of artery and vein-derived endothelium in the mixed population scenario. (i) Schematic representation of the three identity scenarios in dorsal part of

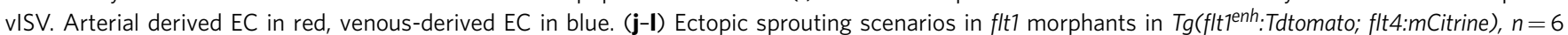
embryos. (j) Ectopic venous sprout devoid of flt $7^{\text {enh }}$ expressing artery-derived EC (arrowhead). (k) Ectopic venous sprout (arrowhead) containing both

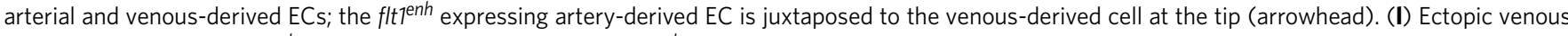
sprout only containing flt $7^{\text {enh }}$ expressing artery-derived ECs; flt $7^{\text {enh }}$ (red) and flt4 (green) were expressed by the same cell which appears in yellow (arrowhead). (m-o) Schematic representation of the three ectopic venous sprouting scenarios. (p) Ectopic sprouting upon loss of flt7 in $T g\left(f / t p^{e n h}\right.$ : $T d t o m a t o ;$

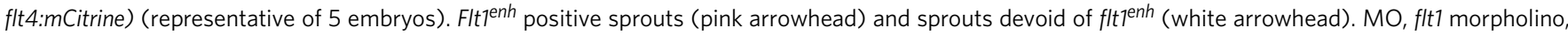
1ng. alSV, arterial intersegmental vessel; DA, dorsal aorta; DLAV, dorsal longitudinal anastomotic vessel; PCV, posterior cardinal vein; vISV, venous intersegmental vessel. Scale bar, $20 \mu \mathrm{m}$ in $\mathbf{j}-\mathbf{I}, 10 \mu \mathrm{m}$ in $\mathbf{b}-\mathbf{f}, \mathbf{p}$. 

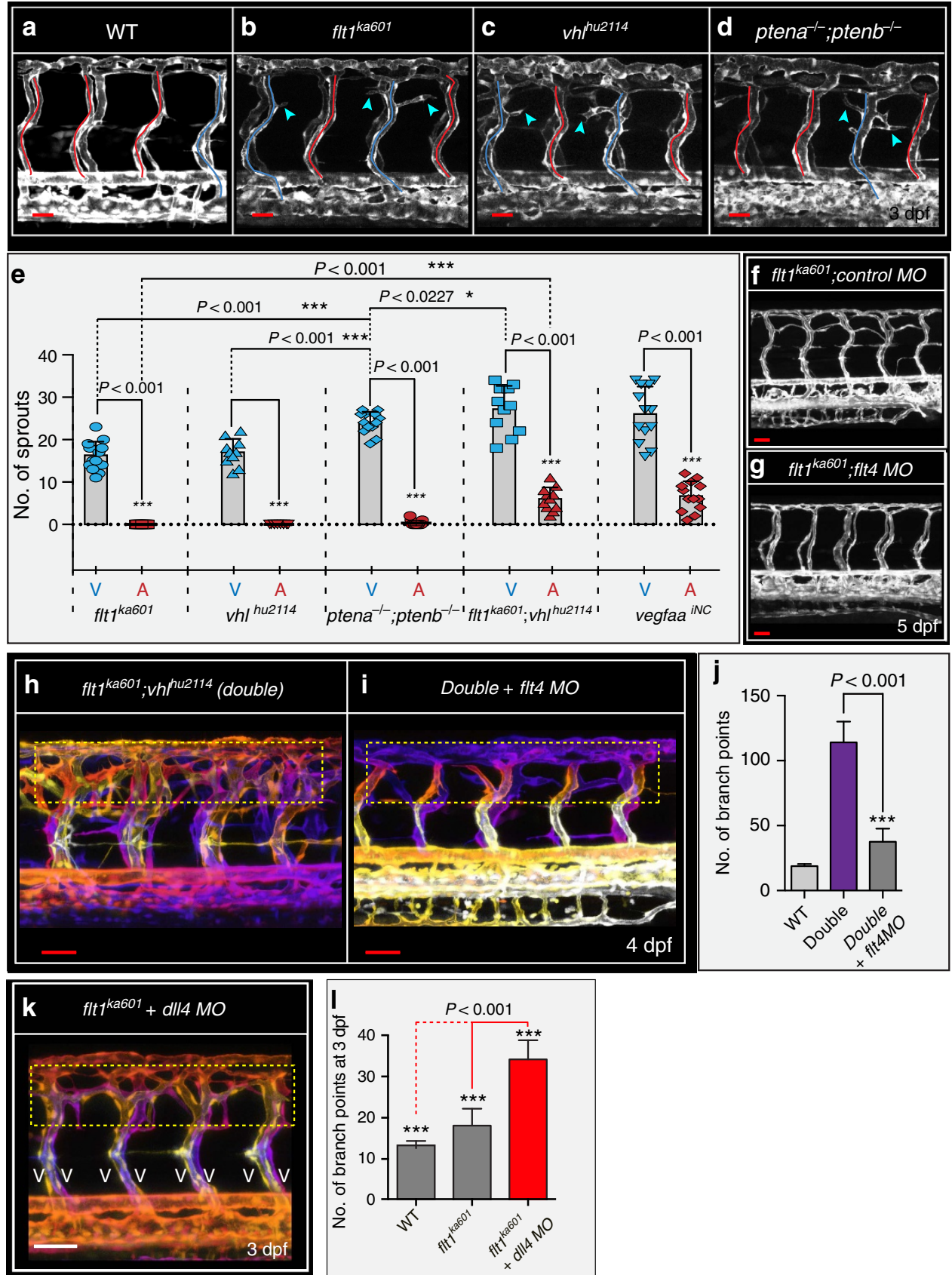

Figure 5 | fltt $\mathbf{k}^{\mathbf{k a 6 0 1}}$ mutants and vegfaa gain-of-function scenarios promote ectopic venous sprouting. (a-d). Trunk vasculature at $3 \mathrm{dpf}$ in WT (a), $f \mid t 7^{k a 601}(\mathbf{b}), v h^{h u 2114}(\mathbf{c})$ and ptena ${ }^{-/-} ;$; ptenb ${ }^{-/-}$double mutants (d) in $T g(k d r l: E G F P)^{5843}$ background. Note ectopic sprouts originate from vISVs (blue arrowheads) in mutants. aISVs indicated in red, vISVs in blue. (e) Quantification of ectopic sprouting in indicated mutants and inducible neuronal-specific vegfaa gain-of-function. In all models ectopic sprouting preferentially occurs in veins, mean \pm s.e.m., $n=10-13 /$ per group, $t$-test. (f,g) flt ${ }^{k a 601}$ mutants show

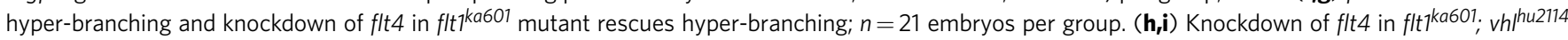
double mutants (double) rescues hyper-branching; compare yellow dotted box in $\mathbf{h}, \mathbf{i}$. The position of vessels is colour-coded. Note: on loss of flt4 the trunk vasculature consists almost exclusively of alSV. (j) Quantification of $\mathbf{h}, \mathbf{i}$. Mean \pm s.e.m., $n=12$ embryos per group, $t$-test. (k,l) Loss of $d I l 4$ in fltt ${ }^{k a 601}$ mutants augments ectopic branching compared with untreated $f(t)^{k a 601}$ mutants. Note: on loss of $d I l 4$ the trunk vasculature consists almost exclusively of vISV; $n=11$ embryos per group, $t$-test. alSV, intersegmental artery; MO, morpholino; vISV, intersegmental vein. Scale bar, $30 \mu \mathrm{m}$ in $\mathbf{a}-\mathbf{d}, \mathbf{f} ; 50 \mu \mathrm{m}$ in $\mathbf{f}-\mathbf{i}, \mathbf{k}$.

and activate downstream Kdrl signalling even further. Indeed, $f l t 1^{k a 601} ;$ vhl $l^{h u 2114}$ double mutants showed more severe hyper-branching of the trunk vasculature when compared with single mutants (Fig. 5e,h). Accordingly, flt1 ${ }^{k a 601} ;$ vhl $l^{\text {hu2114 }}$ double mutants developed more ectopic venous sprouts when compared with either single $f l t 1^{k a 601}$ or single $v h l^{h u 2114}$ mutants (Fig. 5e). The $f l t 1^{k a 601}$; $v h l^{h u 2114}$ double mutants also developed a small number of ectopic arterial sprouts after $2.5 \mathrm{dpf}$ (Fig. 5e).
However, venous sprout numbers were three times higher $(P<0.001)$ than arterial sprout numbers at this stage (Fig. 5e). Changes in primary aISV (24hpf) sprouting were not observed (Supplementary Fig. 6e,f,g). Endoxifen-induced neuronal-specific overexpression of vegfaal65 at $52 \mathrm{hpf}$ in WT embryos also promoted ectopic venous sprouting (Fig. 5e; Supplementary Fig. 8e,f). In addition, a smaller number of ectopic arterial sprouts was noted, similar to $\mathrm{flt}^{\mathrm{ka601}}$; vhl $\mathrm{hu}^{\mathrm{hu} 114}$ 
double mutants (Fig. 5e). Taken together, ectopic venous sprouting was conserved in five vegfaa gain-of-function scenarios.

Ectopic sprouting in flt $^{k a 601}$ mutants requires veins. To prove that in $f l t 1^{k a 601}$ mutant sprouts indeed emanated from veins, we interfered with early arterial-venous remodelling by blocking flt4 (ref. 26) (Fig. 5f,g; Supplementary Fig. 6l). Loss of flt4 in flt $1^{k a 601}$ mutants interfered with arterial-venous remodelling; as a consequence almost all trunk ISVs remained arterial ${ }^{26}$ (Supplementary Fig. 61). In line with the requirement for veins, the $f l t 1^{k a 601}$ hyper-branching phenotype was rescued (Fig. 5f,g). Furthermore, flt4 loss-of-function in the $\mathrm{flt}^{\mathrm{ka601}}$; $v \mathrm{l}^{\text {hu2114}}$ double mutants (denoted as double in Fig. 5h,i) also significantly reduced branching complexity (Fig. 5h-j; method quantification of branch points in Supplementary Fig. 1n). As ectopic sprouting requires venous endothelium, we next reasoned that promoting vISV formation in $f t 1^{k a 601}$ mutants should augment branching. Vessel identity and Notch signalling are linked. In zebrafish, it is established that loss of the Notch ligand Dll4 promotes venous cell fate and dll4 loss-of-function embryos display a trunk vasculature consisting almost exclusively of $\mathrm{vISVs}^{45}$. Accordingly, loss of dll4 in flt $1^{k a b 01}$ mutants significantly augmented ectopic branching when compared with control flt1 ${ }^{k a 601}$ mutants (Fig. 5k,1).

Notch, pericytes and ectopic venous sprouting in $\boldsymbol{f l t}^{\mathbf{k a 6 0 1}}$. One explanation for the low arterial responsiveness in vegfaa gain-of-function scenarios may involve high arterial Notch activity since Notch acts as a repressor of sprouting in arteries, downstream of Vegfaa signalling ${ }^{25,35,46}$. To inhibit endothelial Notch signalling in arterial ISVs of $f t 1^{k a 601}$ mutants, we expressed a dominant negative form of the Notch co-activator MAML (DN-MAML-EGFP) in an endoxifen inducible manner (Fig. $6 \mathrm{a}, \mathrm{b})^{47}$. We used the flt $1^{\text {enh }}$ promoter construct which is mainly active in aISVs (ref. 39) to drive gal4-ERT2;UAS:DN-MAML-eGFP (notch ${ }^{\mathrm{i} \triangle \mathrm{EC}}$ ) in $f t 1^{k a 601}$ mutants. Transgene expression was initiated at $52 \mathrm{hpf}$ by adding endoxifen. Endothelial-specific DN-MAML gain-offunction in $f t 1^{k a 601}$ mutants induced ectopic aISV sprouting at the level of the neural tube (Fig. 6a,b,f). Even more pronounced ectopic arterial sprouting was observed with the $\gamma$-secretase inhibitor LY-411575 that blocks Notch activation; adding LY-411575 at $2 \mathrm{dpf}$ activated ectopic arterial sprouting in flt $^{\text {ka601 }}$ mutants (Fig. 6c-f). Venous sprout numbers were not significantly changed upon DN-MAML $(16.1 \pm 3.45$ versus $17.1 \pm 2.88)$ or LY-411575 treatment $(15.9 \pm 2.89$ versus $14.2 \pm 1.69$ ). Addition of LY-411575 to WT at $2 \mathrm{dpf}$ had no effect. To explain differential AV responsiveness, we also considered differences in pericyte cell coverage (Fig. 6g-j). Overall, pericytes were scarce with $88 \%$ of all ISVs investigated not being covered by pericytes. In the remaining $12 \%$ of cases, pericytes were found in both aISVs $(9.94 \%)$ and vISVs (1.91\%) along the ISV ventral-dorsal axis. In the most dorsal aspect of aISV and vISV, the region where ectopic sprouting occurs in

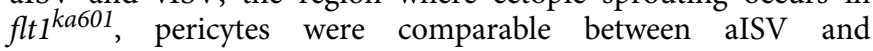
vISV (2.48\% and $1.91 \%$ respectively, Fig. $6 \mathrm{k})$.

Vegf and Flt1 determine extent of spinal cord vascularization. Neurons expressed vegfaa (Supplementary Fig. 1), and neuronal cells of both $3 \mathrm{dpf}$ WT and vhl loss-of-function embryos had significantly higher vegfaa levels than non-neuronal cells (Fig. 7a,b; FACS settings in Supplementary Fig. 7a-d). Furthermore, neuronal vegfaa expression was significantly increased in $v h l$ loss-of-function when compared with WT (Fig. 7a,b). Thus, at this stage of development neurons are the major source of vegfaa, and not other tissues like developing muscle ${ }^{48}$. We next examined whether neurons can direct sprouts into the neural tube (Fig. $7 \mathrm{c}-\mathrm{i}$ ). We compared the flt $^{k a 601} ;$ vhl hu2114 double mutant (Fig. 7c,d) with flt $1^{k a 601}$ mutant and WT and found striking changes in optical sections of the neurovascular interface (Fig. $7 \mathrm{e}-\mathrm{h}$ ). In flt $^{\mathrm{ka} 601}$, sprouts occasionally projected into the neural tube (Fig. $7 \mathrm{~g}$ ), whereas in $f l 1^{k a 601} ; v h l^{h u 2114}$ double mutants many branches invaded the neural tube (Fig. 7 h,i).

In the mutants with vegfaa gain-of-function, the spinal cord becomes vascularized relatively early, between 3 and $4 \mathrm{dpf}$. In WT, the spinal cord is vascularized much later in development starting in the period between 12 and $14 \mathrm{dpf}$ (Fig. 7j-1). In those older WT embryos, sprouts preferentially emanated from venous ISVs, displayed nuclear positioning as described for the flt1 mutant (Supplementary Fig. 7e,f) and the onset of vascularization of the WT spinal cord coincides with decreased sflt1 expression during this stage of development (Supplementary Fig. $7 \mathrm{~g})$.

Neuronal sFlt1 and Vegfaa regulate sprouting from veins. We next generated tissue-specific and inducible $f t 1$ and vegfaa gainof-function models. Loss of neuronal sFlt1 in flt ${ }^{k a 601}$ mutants may augment neuron-derived Vegfaa availability and promote ISV sprouting. Hence, restoring neuronal sFlt1 in flt $1^{k a 601}$ mutants should provide a rescue, whereas neuronal-specific flt1 loss-of-function should induce hypersprouting. To test the first scenario we expressed -3.2elavl3:gal4-ERT2;UAS:GFP-p2A-sflt1 $\left(s f t 1^{i N C}\right.$ ) in flt $1^{\text {ka601 }}$ mutants (Fig. 8a-d, branch quantification method in Supplementary Fig. 1n). This construct allows precise time-controlled expression of sflt 1 specifically in neurons. We found that transgene activation in neurons at $52 \mathrm{hpf}$, just before the emergence of the ectopic sprouts in $f t t 1^{k a 601}$ mutants, rescued the vascular hyper-branching phenotype (Fig. $8 \mathrm{~b}-\mathrm{d}$ ).

We next explored whether neuron-specific loss of flt1 is sufficient to induce ISV hyper-branching (Fig. 8e-i). To accomplish neuron-specific loss of flt 1 we expressed the flt1 targeting $s g R N A^{f l t 1 E 3}$ (U6:sgRNA ${ }^{f t 1 E 3}$, the same sgRNA as used to generate $f t t^{k a 601}$ mutants; expressed in all cells) together with the Cas9 construct employing the Gal4UAS system under the control of the pan-neuronal promoter

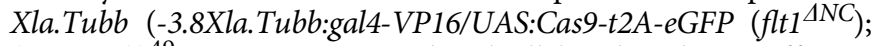
(Fig. 8e) $)^{49}$. To optimize the biallelic knockout efficacy, we injected the construct into embryos heterozygous for the $f t t 1-1 \mathrm{nt}$ allele $\left(f t 1^{\mathrm{ka} 601 /+}\right)$. GFP signal was detected in spinal cord neurons indicating efficient $X l a$.Tubb-driven neuron-specific expression of Cas 9 (Fig. 8h). Neuronal loss of flt1 significantly induced ectopic venous sprout formation when compared with WT and $f l t 1^{\text {ka601/+ }}$ heterozygous mutants (Fig. 8f-i). In contrast, sprouting was not observed when $\operatorname{Cas} 9$ was expressed under a vascular promoter (Supplementary Fig. 8a) or in embryos only carrying the sgRNA without Cas9.

To substantiate the contribution of neuronal sflt 1 we next employed multiplexed custom designed miRNAs directed against sflt1 $3^{\prime}$ UTR arranged with a common miR-155 backbone ${ }^{50}$ (Supplementary Fig. 8b). The constructs were expressed under control of vascular $\left(f_{t} t^{e n h}\right)$ and neuronal (Xla.Tubb) specific promoters. Targeting neuronal sflt1 resulted in ectopic sprouting (Supplementary Fig. 8c), but targeting vascular sflt1 failed to induce sprouts (Supplementary Fig. 8d).

Next we performed cell transplantation experiments, which demonstrated that neuronal flt 1 and not vascular flt 1 is the physiologically relevant mediator of sprouting at the level of the neural tube (Fig. 8j-1). Transplantation of flt 1 mutant neurons into WT hosts induced ectopic sprouting (Fig. 8k). 

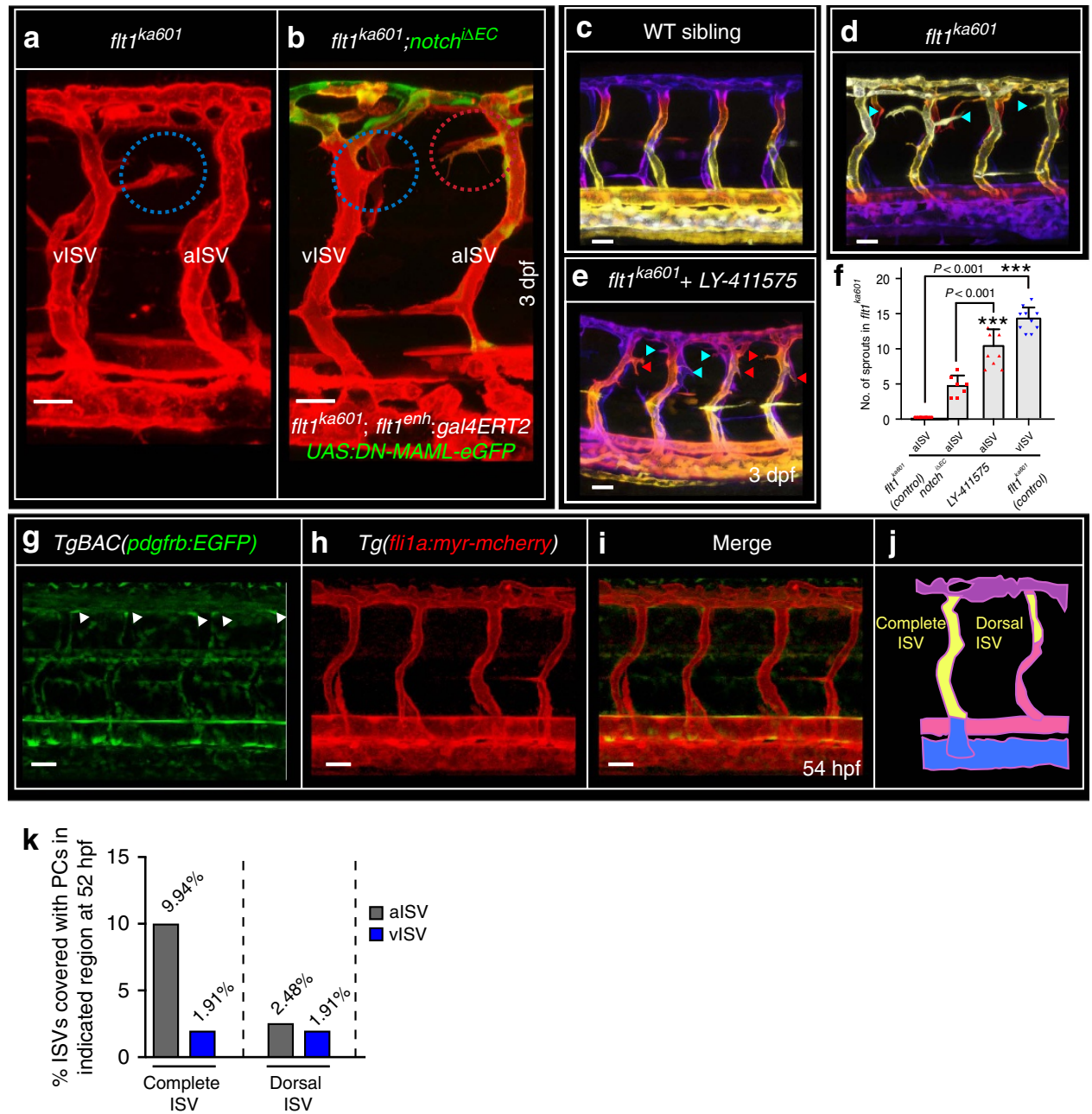

Figure 6 | Notch inhibits ectopic arterial sprouting in fltt $\mathbf{1}^{\mathbf{k a 0 0}}$ mutants. (a), flt $7^{\mathrm{ka601}}$ mutants show ectopic venous sprouts (blue circles), but no arterial sprouts. (b) Inhibiting arterial Notch by endoxifen-induced arterial ISV-specific expression of dominant negative MAML-eGFP (Notch ${ }^{\mathrm{I}} \mathrm{ECC}$ ) at $52 \mathrm{hpf}$ under control of the flt $7^{\text {enh }}$ promoter in $f_{1 t} 7^{\mathrm{ka} 601}$ mutant results in the emergence of ectopic arterial sprouts (red circles); representative image from 7 experiments. (c-e) Trunk vasculature of WT (c), flt $7^{k a 601}(\mathbf{d})$ and flt $7^{k a 601}$ treated with Notch inhibitor LY-411575 (e). LY-411575 was added at 2 dpf. Note the emergence of ectopic arterial sprouts upon LY-411575 treatment. (red arrowhead: arterial sprout; blue arrowhead: venous sprout). (f) Quantification of experiments in (a-e), mean \pm s.e.m., $n=7$ for Notch ${ }^{i \Delta E C}, n=10$ for $L Y-41157$ treatment, $n=10$ for flt $7^{k a 601}$; $t$-test. (g-i) Imaging of pericytes in

TgBAC(pdgfrb:EGFP); Tg(fliTa:myr-mcherry) double transgenic at $54 \mathrm{hpf}$. (j) Schematic representation of pericyte number counting in ISVs as performed in (k). (k) Quantification of pericyte recruitment in aISVs and vISVs at $54 \mathrm{hpf}$. ( $n=246$ ISVs from 14 embryos). ISV, intersegmental artery; Notch i ${ }^{\Delta E C}$, inducible ISV-specific loss of Notch; vISV, intersegmental veina. Scale bar, $25 \mu \mathrm{m}$ in a-e,g-i.

In contrast, transplantation of flt 1 mutant endothelial cells into WT hosts failed to induce sprouting (Fig. 81).

To prove that neuron-derived Vegfaa promotes hyper-branching, we generated neuronal tissue-specific and inducible vegfaa165 gain-of-function zebrafish (Supplementary Fig. 8e,f; quantification in Fig. 5e). Transgenic expression was initiated by adding endoxifen after completion of AV remodelling at $52 \mathrm{hpf}$. In this scenario hyper-branched neovascular networks formed at the level of the neural tube, similar to flt $1^{k a 601}$ mutants (Supplementary Fig. 8e,f). Neuronal vegfaa121 was also capable of inducing sprouting (Supplementary Fig. 8g). In contrast, neuron-specific and inducible vegfc gain-of-function, induced at $54 \mathrm{hpf}$, did not induce ectopic sprouts (Supplementary Fig. 8h). Timing of transgene expression was relevant as inducible neuronspecific vegfaal65 overexpression prior to completion of AV remodelling resulted in thickened abnormal vascular structures (Supplementary Fig. 8i,j). In the same line, neuronspecific constitutive overexpression of $s$ flt 1 completely annihilated ISV formation (Supplementary Fig. 8k).
To confirm that the $f t 1^{k a 601}$ phenotype involved gain of Vegfaa, we titrated vegfaa levels using a low dose vegfaa targeting morpholino ${ }^{51}$. Reducing vegfaa in flt ${ }^{\mathrm{ka} 601}$ mutants rescued the hyper-branching phenotype (Fig. $8 \mathrm{~m}-\mathrm{o}$ ). Vegfaa signals via Kdrl and application of ki8751, an established Kdrl tyrosine kinase inhibitor in zebrafish ${ }^{52}$ to $f t 1^{k a 601}$ mutants at $2.5 \mathrm{dpf}$ annihilated the formation of the ectopic neovascular networks (Supplementary Fig. 81,m,o). In contrast, the Flt4-specific tyrosine kinase inhibitor MAZ51 (ref. 52) did not rescue hyper-branching in $f t 1^{k a 601}$ (Supplementary Fig. 81,n,o). Vegfaa-driven primary artery sprouting can occur in the absence of blood flow perfusion. To test if Vegfaadriven ectopic venous sprouting in $f t 1^{k a 601}$ mutants is affected by blood flow, we modulated cardiac activity and flow with $\mathrm{BDM}$ or tricaine. We found that loss of flow completely rescued ectopic hyper-sprouting in $f l 1^{k a 601}$ mutants (Supplementary Fig. 9a-e). Inhibition of PI3 kinase with wortmannin also significantly reduced ectopic sprouting (Supplementary Fig. 9f). 

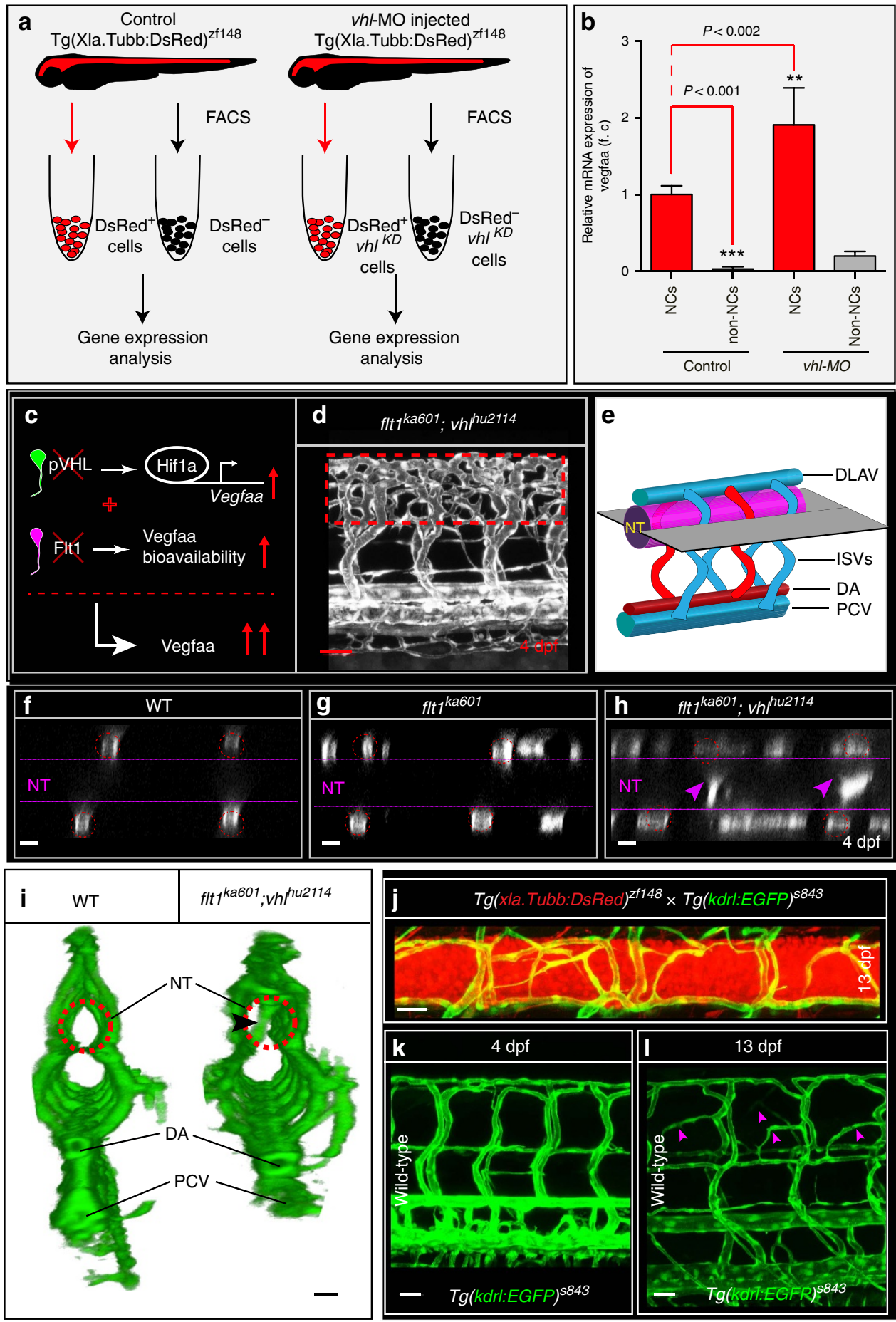

Figure 7 | Neurons are a major source of Vegfaa and attract sprouting vessels. (a) FACS procedure for obtaining neuronal cells in control and vhl morphants using $\mathrm{Tg}$ (Xla.Tubb:DsRed $)^{2 f 148}$ neuronal reporter embryos. (b) Quantification of vegfaa expression using real-time qPCR in FAC-sorted cell populations at $3 \mathrm{dpf}$. Note that neuronal cells expressed significantly more vegfaa than non-neuronal cells. Loss of vhl promoted neuronal vegfaa expression. Mean \pm s.e.m., $n=3$ separate experiments in triplicate (two-way ANOVA). (c) Schematic representation: loss of vhl augments vegfaa transcription, loss of flt1 augments Vegfaa bioavailability; combining both mutants augments Vegfaa bioavailability above single mutant level. (d) Trunk vasculature in $\mathrm{flt}^{\mathrm{ka} 601}$; $\mathrm{vh} \mathrm{I}^{\text {hu2114 }}$ double mutants at $4 \mathrm{dpf}$. Note the severe hyper-branching at the level of the neural tube, red-dotted box. (e) Schematic representation of optical section (shown in $\mathbf{f}-\mathbf{h}$ ) through the neural tube and associated trunk vasculature. (f-h) Dorsal view on optical section through WT (f), flttika601 (g) and flt ka601; $^{\text {vh }}$ h $^{\text {hu2114 }}$ double mutants (h). Note invasion of sprouts into the neural tube in double mutants (arrowheads in $\mathbf{h}$ ). Red circle indicates position of ISVs, dotted line neural tube boundary. (i) Transverse 3D-rendered view of vasculature (green) through the trunk in WT (left panel) and $\mathrm{flt}^{\mathrm{ka601}} \mathrm{vh}^{\text {hu2 } 214}$ double mutants (right panel); note vessels penetrating the neural tube in mutant (compare vessel in dotted circle right panel, arrowhead; such vessels are absent in WT left panel; representative image from 3 separate experiments). (j) Representative image of spinal cord vascular network in $\mathrm{Tg}$ (xla.Tubb:DsRed) ${ }^{2 f 148} ; \mathrm{Tg}(\mathrm{kdrl}: \mathrm{EGFP})^{\mathrm{s} 843}$ double transgenic at $13 \mathrm{dpf}$. (k, I) Comparison of trunk vasculature in WT at $4 \mathrm{dpf}(\mathbf{k})$ and at $13 \mathrm{dpf}(\mathbf{I})$; note the emergence of ectopic branches (pink arrowheads) at level of the spinal cord. DA, dorsal aorta; f.c. fold change; KD, knockdown; NT, neural tube; NC, neuronal cell; PCV, posterior cardinal vein. Mutants are in Tg(kdrl:EGFP) 5843 background. Scale bar, $50 \mu \mathrm{m}$ in d; $25 \mu \mathrm{m}$ in $\mathbf{f - I}$. 

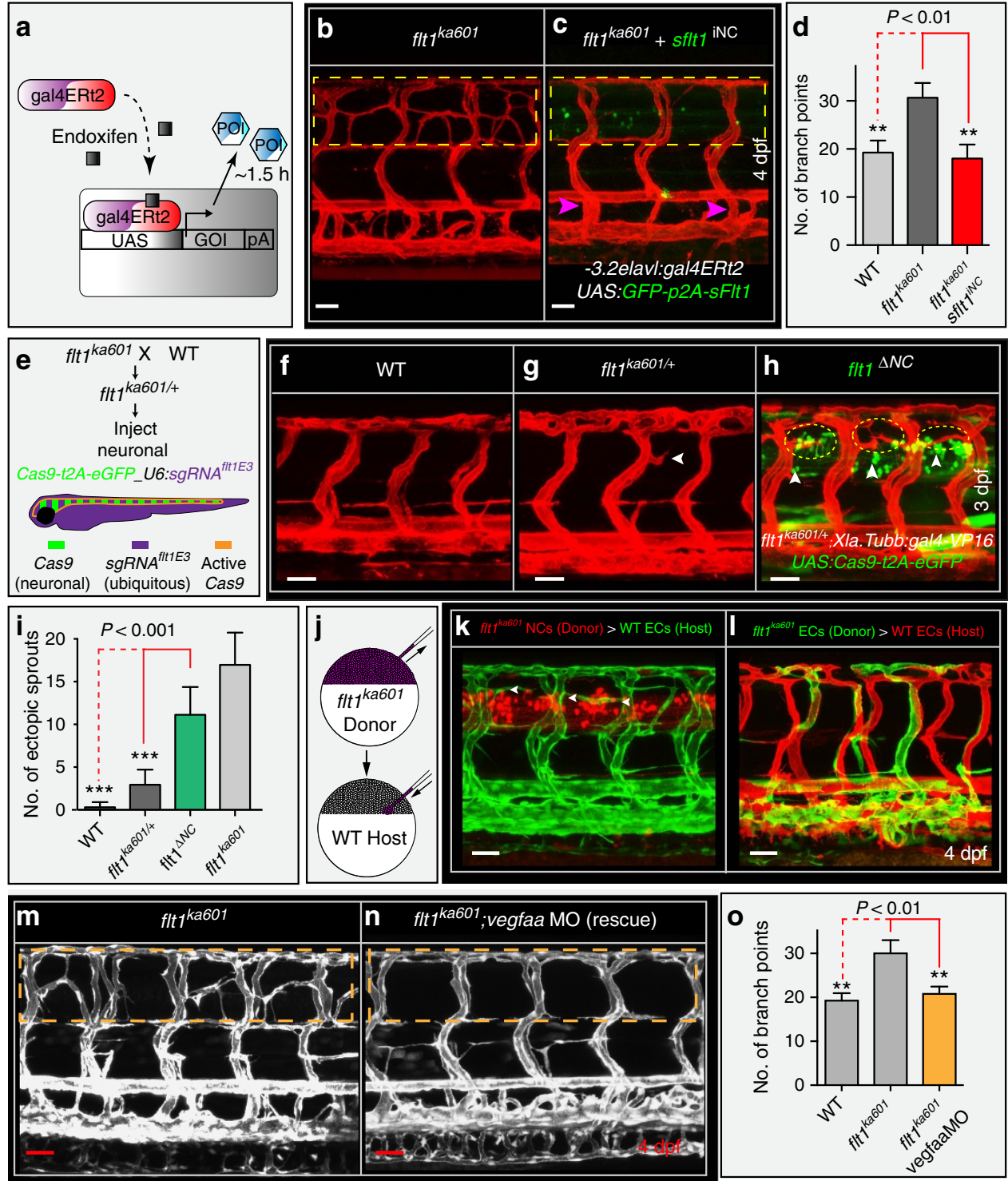

Figure 8 | Neuronal Flt1 regulates vascular branching by titrating neuronal Vegfaa. (a) Schematic representation of endoxifen inducible gain-of-function approach in zebrafish. In the present situation Gal4 is under the control of neuron-specific promoters elavl or Xla.Tubb. Expression can be observed within $1.5 \mathrm{~h}$ upon endoxifen application. (b) Hyper-branching in flt $7^{\mathrm{ka} 601}$ mutants (dotted box). (c) Endoxifen inducible neuron-specific sflt gain-of-function rescues hyper-branching in flt $7^{\mathrm{ka} 601}$ mutants; compare dotted box in c and b. Purple arrowheads indicate vISVs; endoxifen was applied at $52 \mathrm{hpf}$. (d) Quantification of rescue in (b,c), mean \pm s.e.m, $n=15-19$ embryos per group. (e) Approach for generating a neuron-specific flt1 mutant. Cas 9 was expressed under control of neuronal promoter Xla.Tubb; sgRNA was expressed ubiquitously, resulting in Cas9 activity in neuronal cells only (domain marked by orange border). Heterzygous $f\left(t 7^{k a 601 /+}\right.$ were used to facilitate biallelic knockout. (f-h) Neuron-specific loss of $f(t)$ ( $f\left(t 7^{\Delta N C}\right.$ ) induces ectopic sprouting (h), sprouts in yellow dotted ellipse, arrowheads indicate neuronal cells with Cas9 expression. (i) Quantification of ectopic sprouting for indicated genotypes. Note that neuron-specific loss of flt1 significantly augments ectopic sprouting (green bar) mean \pm s.e.m, $n=16$ embryos per group, $t$-test. (j-I) Transplantation of flt 1 mutant neuronal cells $(\mathbf{k})$ and endothelial cells (I) into WT. Note: transplantation of flt 1 mutant neuronal cells induced sprouting (k, arrowheads); 9 out of 12 neuronal cell transplantations resulted in sprout formation. In all 10 endothelial cell transplantations, sprouts were absent (I). (m,n) Low dose morpholino-mediated reduction of vegfaa expression in flt ${ }^{k a 601}$ mutants rescues sprouting defects; compare dotted box in $(\mathbf{m}, \mathbf{n})$. (o) Quantification of rescue in $(m, n)$, mean \pm s.e.m., $n>5$ per group, $t$-test. DA, dorsal aorta; PCV, posterior cardinal vein; DLAV, dorsal longitudinal anastomotic vessel; NT, neural tube. GOI, gene of interest; POI, protein of interest; iNC, inducible, neuronal cell specific gain-of-function; $\triangle N C$, neuronspecific loss of flt7; MO, morpholino. Scale bar, $50 \mu \mathrm{m}$ in $\mathbf{b}-\mathbf{h}, \mathbf{m}, \mathbf{n} ; 25 \mu \mathrm{m}$ in $\mathbf{k}, \mathbf{l}$.

\section{Discussion}

Intimate cross-talk between vessels and the nervous system is important for tissue homeostasis. During embryonic development, neuronal stem cells differentiate into mature neurons, a process that associates with a change in cellular metabolism ${ }^{53}$. Concomitantly with developmental neurogenesis, changes occur in the vascular network feeding the spinal cord. We show in the zebrafish embryo that neurons in the developing spinal cord express the pro-angiogenic ligand Vegfaa and antiangiogenic soluble Vegf receptor-1, sFlt1, which acts as a Vegfaa scavenger (Fig. 9). Spinal cord neurons are in close contact to the developing trunk vasculature, and we show that these vessels are responsive to changes in neuronal sFlt1 and Vegfaa. Using a combination of global and tissue-specific 


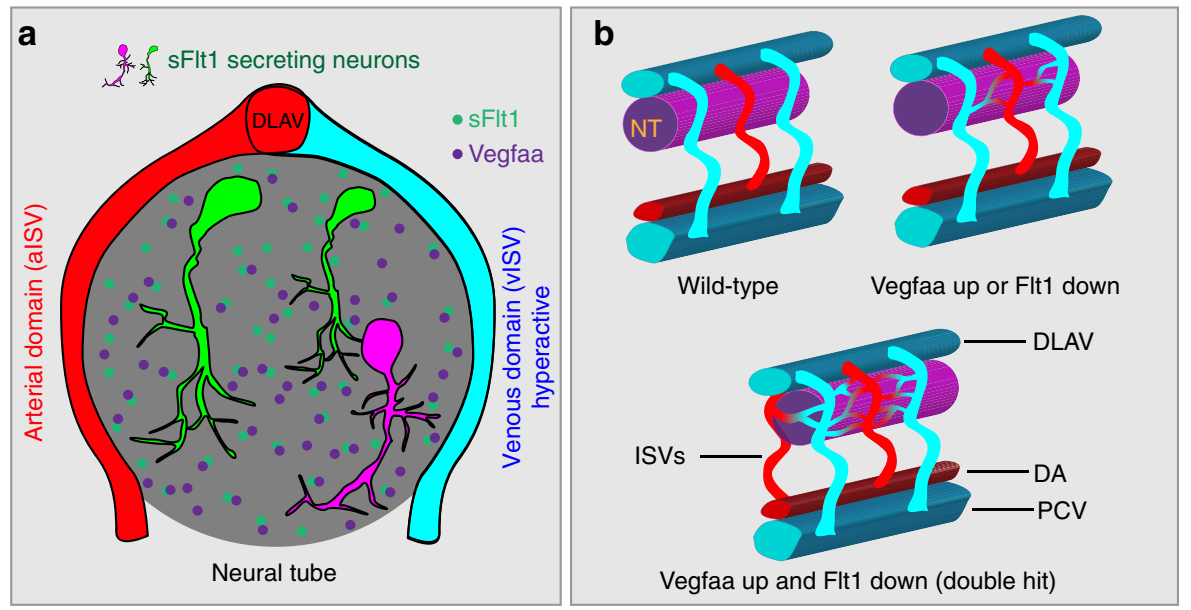

Figure 9 | Schematic representation of neurovascular communication involving neuronal sFlt1-Vegfaa and sprouting from intersegmental veins.

(a) Spinal cord neurons produce both sFlt1 and Vegfaa in close proximity to the dorsal aspect of intersegmental arteries and veins. (b) Schematic

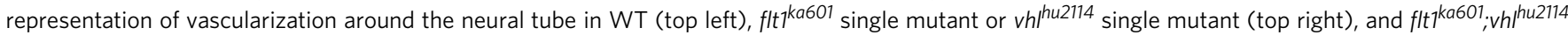
double mutant (bottom). Loss of flt7 or vhl induces the formation of a peri-neural tube network, and combining both mutants in addition promotes sprouting into the neural tube. NT, neural tube; ISV, intersegmental vessel (a-arterial, v-venous); DA, dorsal aorta; PCV, posterior cardinal vein; DLAV, dorsal longitudinal anastomotic vessel; hpf, hours post fertilization.

loss of flt 1 mutants, and further substantiated by vegfaa loss- and gain-of-function experiments as well as cell transplantations, we demonstrate that neuronal sFlt1 restricts neuronal Vegfaa and vessel branching morphogenesis at the neurovascular interface. Differential regulation of vegfaa and sft 1 allows orchestration of the onset and extent of spinal cord vascularization (Fig. 9). We propose that neurons may use sFlt1-Vegfaa to adjust vascularization according to their developmental needs.

Flt1 ${ }^{\text {ka601 }}$ mutants develop ectopic sprouts emanating from venous ISVs around embryonic day 2.5. Neuron-specific targeting of $f l t 1$ or $s f l t 1$ using CRISPR/Cas9- and miRNA-based approaches respectively, result in $f t 1^{k a 601}$ comparable phenotypes. Transplantation of flt1 mutant neurons into WT hosts induces ectopic sprouting which is not observed after transplantation of flt 1 mutant endothelial cells, suggesting that neuronal $f l t 1$ is the physiologically relevant mediator in our mutant. Neuronal-specific gain of $s f l t 1$, reducing vegfaa levels, or inhibition of Kdrl signalling provide a rescue suggesting that ectopic venous sprouting is mediated by the VegfaaKdrl signalling axis. Accordingly, ectopic venous sprouting from the dorsal aspect of vISVs is conserved in five independent vegfaa gain-of-function scenarios.

Previous studies have shown that during AV remodelling, aISV-derived endothelial cells remain integrated in the dorsal aspect of vISVs (ref. 39). We confirm that remodelled artery-derived endothelial cells indeed contribute to this domain although they are not the sole or most important endothelial source. Using in vivo cell tracking we find posterior cardinal vein-derived endothelial cells migrating against the direction of blood flow to populate venous ISVs including the dorsal aspect where sprouts are formed. Here, venous-derived endothelium can co-exist with the artery-derived endothelium. With respect to the endothelial cells populating the dorsal part of vISVs, our data now reveal three different scenarios. The dorsal aspect can contain a mix of both artery and venous-derived endothelium (43\%), only vein-derived endothelium (48\%) or only artery-derived endothelium (8\%). On loss of flt1, both artery- and vein-derived endothelium give rise to ectopic vISV sprouts. This prompts toward the concept that integration into the local venous ISV environment constitutes a permissive factor for sprouting, regardless of the endothelial origin.
Flt $1^{\text {ka601 }}$ mutants display ectopic sprouting in vISVs but not in aISVs, indicating that AV vessel identity or compartmentspecific cues may be involved in the novel sprouting type described here. Notch is tightly linked to both AV vessel specification and sprouting, as Notch programs arterial identity and Notch signalling represses sprouting of arteries ${ }^{25,45}$. Lack of Notch associates with venous identity and Notch signalling is absent in venous ISVs (ref. 35). Interference with AV remodelling or Notch indeed affects sprouting numbers in $f t 1^{k a 601}$. Inhibiting the remodelling of arterial ISVs into venous ISVs in $f l 1^{k a 601}$, and thus creating a trunk vasculature that almost exclusively consists of aISVs, rescues ectopic hypersprouting. Conversely, promoting venous cell fate and creating a trunk that consists of vISVs by knock-down of dll4 augments vascular branching in $f t 1^{k a 601}$. Inhibiting Notch by endothelial expression of DN-MAML or pharmacological treatment with LY-411575 stimulate ectopic arterial sprouting in $f l 1^{k a 601}$, without affecting venous sprout numbers. Thus active Notch signalling in arteries most likely accounts for the observed AV sprouting differences. We propose that the artery-derived endothelial cells that become incorporated in vISVs on $\mathrm{AV}$ remodelling are relieved from Notch and adopt a venous fate, enabling them to respond to changes in local Vegfaa.

Our data show that vISVs contribute to the vascularization of the spinal cord with Vegfaa-Kdrl signalling mediating vISV branching events. Interestingly, in other domains of the zebrafish trunk venous vasculature, endothelial cells are responsive to alternative signals. The Vegfc-Flt4 signalling pathway drives secondary venous sprouting from the PCV contributing to the formation of the lymphatic vasculature. BMP-Smad signalling has been shown to promote venous sprouting in the caudal vein plexus $^{54}$. Furthermore, PCV-derived endothelial cells contribute to the formation of the gut vasculature ${ }^{55}$. Regeneration of the tail fin vasculature upon injury also starts from the venous side ${ }^{56}$. The concept emerging from these observations is that initiation of organ vascularization is initiated or proceeds from the venous vasculature. The mouse embryo coronary vasculature is vein-derived, and coronary arteries form by developmental reprogramming of venous endothelium ${ }^{57}$. Context-dependent heterogeneity in signalling mechanisms establishing venous branching, may allow versatile control of tissue vascularization in a spatio-temporal manner. 
Using sFlt1 as a rheostat to control Vegfaa bioavailability, constitutes a means to regulate Vegfaa independent of vegfaa promoter activity, vegfaa mRNA or protein stability. We propose that this enables neurons to dynamically fine-tune the extent and onset of peri-neural vascular network formation and sprouting into the spinal cord. While the peri-neural network may serve to sustain growth of the developing nervous system, vessel sprouting into the spinal cord and relief of hypoxia has been associated with changes in neuronal stem cell metabolism, triggering differentiation events ${ }^{53}$. Therefore, untimely or excessive vascularization of the spinal cord is potentially harmful as it may promote premature stem cell differentiation and disrupt the carefully orchestrated neuronal specification process. We propose a two-tiered checkpoint mechanism involving sFlt1 and Vegfaa, requiring two decisions to guide vascularization, namely Vegfaa up- and sFlt1 downregulation, to protect neurons from harmful angiogenesis and oxygen variations during early stages on the one hand, and on the other hand to enable more mature neurons to attract sufficient vessels into the spinal cord after stem cell differentiation has been completed.

\section{Methods \\ Ethics statement. Zebrafish husbandry and experimental procedures were performed in accordance with the German animal protection standards and were approved by the government of Baden-Württemberg, Regierungspräsidium Karlsruhe, Germany (Akz.: 35-9185.81/G-93/15).}

Transgenic lines. $T g(f l i 1 a: E G F P)^{y 1}, T g(k d r l: h s a .-H R A S-m c h e r r y)^{s 916}, T g B A C$

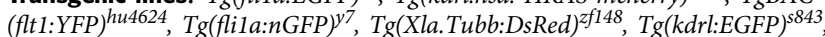

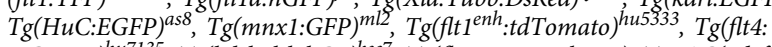
mCitrine $)^{h u 7135}, \mathrm{Tg}\left(\mathrm{kdrl}:\right.$ nlskikGR) ${ }^{h s c 7}, \mathrm{Tg}($ fli1a:myr-mcherry), TgBAC(pdgfrb:EGFP) as well as $v h h^{h u 2114}$ and ptena ${ }^{-/-} ;$ptenb $^{-1+}$ mutants were used as published ${ }^{39,44,58-61}$.

Morpholino injections. Morpholino antisense oligomers (MOs; Gene Tools) were prepared at a stock concentration of $1 \mathrm{mM}$ according to the manufacturer. MOs were injected into the yolk of one-cell stage embryos. We used the flt 4 ATG MO, $5^{\prime}$-CTCTTCATTTCCAGGTTTCAAGTCC- $3^{\prime}(4 \mathrm{ng})$, the $f l t 1$ ATG MO, $5^{\prime}$-ATATCGAACATTCTCTTGGTCTTGC- $3^{\prime}$ ( $1 \mathrm{ng}$ or $\left.3 \mathrm{ng}\right)$, the $v h l$ elil splice MO 5'-GCATAATTTCACGAACCCACAAAAG-3' $(6 \mathrm{ng})$, the vegfaa ATG MO 5'-GTATCAAATAAACAACCAAGTTCAT-3' (0.3 ng), the dll4 MO $5^{\prime}$-TAGGGTTTAGTCTTACCTTGGTCAC-3' $(6 \mathrm{ng})$, and a control MO 5'-CTCTTACCTCAGTTACAATTTATA-3' (10 ng) (refs 18,26,45,51,62).

mRNA injection and generation of transgenic and mutant lines. For the generation of mutants $1 \mathrm{nl}$ of a mixture containing $600 \mathrm{ng} \mathrm{Ll}^{-1}$ capped and

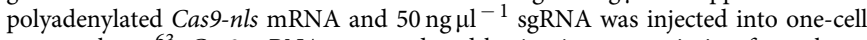
stage embryos ${ }^{63}$. Cas 9 mRNA was produced by in vitro transcription from the MLM3613 plasmid using the mMessage mMachine T7 Ultra Kit (Ambion). The MLM3613 plasmid was a gift from Keith Joung (Addgene plasmid \#42251). For the

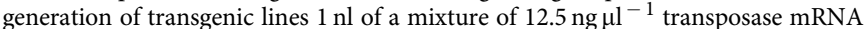
and $25 \mathrm{ng}^{-1} \mathrm{l}^{-1}$ plasmid DNA was injected into one-cell stage embryos.

Cell transplantations. Cell transplantations were performed using $3.5 \mathrm{hpf}$ donor and host blastula-stage embryos. Approximately 50-100 cells were taken from the donor's animal pole and transferred close to the host's lateral marginal zone (for ECs) or slightly above for neuronal cells. Donors and hosts carried distinct neuronal and endothelial-specific reporters to identify the source of ECs and neurons within chimeras.

Generating fIt1 mutants. The zebrafish $f t t 1$ gene consists of 34 exons encoding membrane-bound $f t t 1$ ( $m f l t 1)$ and a shorter soluble flt1 ( $f f t 1)$ form. Soluble flt 1 is generated through alternative splicing of flt 1 mRNA at the exon 10 - Intron 10 boundary (Supplementary Fig. 2a). To annihilate the production of both mflt 1 and sflt 1 and obtain flt 1 mutants, we targeted exon 3, using a CRISPR/Cas approach. We designed five sgRNAs targeting exon 3, encoding the extracellular Igl domain relevant for Vegfaa binding. Oligonucleotides containing the GG-N18 targeting sequence and overhangs were purchased from Eurofins (Ebersberg, Germany). The annealed oligos were ligated into DR274 which was a

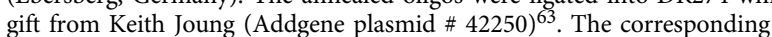
genomic region (surrounding exon 3) was amplified by PCR using primer pair Flt1_E3_gDNA_r and Flt1_E3_gDNA_f and indels were quantified with
T7EI assay or direct Sanger sequencing of the PCR product as described (for primer sequences see Supplementary Table 6) ${ }^{63}$. The T7EI cleavage products of 211 and $249 \mathrm{bp}$ were quantified using ImageJ. The $s g R N A^{\text {ftl } 1 E 3}$ (Supplementary Table 4$)$ with the highest cleavage rate $(\sim 70 \%)$ was used to generate the flt1 mutants. WT embryos were coinjected with sgRNA ${ }^{\text {ft1 } 13}$ plus capped and polyadenylated Cas 9 mRNA. Four independent lines with frame shift mutations were investigated in more detail. The $f t t^{k a 601}$ (exon $3-1 \mathrm{nt}$ allele), flt $1^{k a 602}$ (exon $3-5 \mathrm{nt}$ allele), flt $\mathrm{k}^{\mathrm{ka603}}$ (exon $3+5 \mathrm{nt}$ allele) and $f l t 1^{k a 604}$ (exon $3-14 \mathrm{nt}$ allele) have a premature termination codon (PTC) resulting in a truncated protein devoid of a functional extracellular Vegfaa binding domain. Embryos carrying the mutation were raised and outcrossed to vascular and neuronal reporter lines $\left(\mathrm{Tg}(\mathrm{kdrl}: e G F P)^{\text {s843 }}, \mathrm{Tg}(\mathrm{flila}: \mathrm{e} G F P)^{y 1}\right.$,

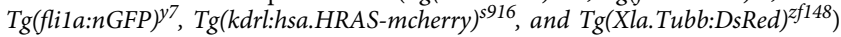

Generation of $\boldsymbol{m f l t} 1$-specific mutants. To generate $m f l t 1$ mutants we used a CRISPR/Cas approach and designed an sgRNA targeting E11b, the first specific $m f l t 1$ exon $^{18}$. In this scenario splicing of intron 10 and exon 1la relevant for generating sflt1 mRNA remains unaffected. Oligos Flt1E11_O1_A_15 and Flt1E11_O2_A_15 were annealed and cloned into DR274 as described for flt1 mutants. Founders were identified by PCR and subsequent Sanger sequencing, using primers Flt1E11A2386576F and Flt1E11A2386151R. We identified four frame shift mutants harbouring a PTC in exon 11b. Flt1 ${ }^{k a 605}$ (exon 11b $+28 \mathrm{nt}$ ), $f_{l t} 1^{k a 606}$ (exon $11 \mathrm{~b}+20 \mathrm{nt}$ ), flt $1^{\text {ka607 }}$ (exon $11 \mathrm{~b}-1 \mathrm{nt}$ ) and $f t 1^{k a 608}$ (exon $11 \mathrm{~b}$ $-1 \mathrm{nt}$ and one mutation) mflt 1 mutants were outcrossed to $T g(k d r l: E G F P)^{s 843}$ and $\mathrm{Tg}(\mathrm{Xla} \text {.Tubb:DsRed })^{z f 148}$. All four $\mathrm{mflt} 1 \mathrm{mutan}$ ts were phenotypically comparable and in this manuscript only the mflt1 mutant $f t 1^{k a 605}$ is shown. All sgRNA sequences and oligos used for annealed oligo cloning into DR274 are listed in Supplementary Table 4,5.

Generation of p5E entry clones. The NBT_tauGFP plasmid was a kind gift by Enrique Amaya. The $3.8 \mathrm{~kb}$ regulatory element derived from neural specific beta tubulin was removed from the NBT_tauGFP using SalI and HindIII and subcloned into SalI and HindIII digested and dephosphorylated p5E_MCS (ref. 64). The $1 \mathrm{~kb}$ flt1 enhancer/promoter fragment from the pMiniTol2_flt1_ECR5a_pro_181_YFP (ref. 39) construct was subcloned into $p 5 E \_M C S$ using KpnI and HindIII. The resulting plasmids were named $\mathrm{p} 5 \mathrm{E}_{-} X l a . T u b b-3.8$ and $p 5 E_{-} f t 1^{\text {enh }}$

Generation of a universal p2A-GFP middle entry clone. To easily detect transgenic cells the pME_eGFP (\#455) from the Tol2kit (ref. 64) was modified by site-directed mutagenesis PCR. The p2A sequence was added before the stop codon of GFP using $p M E_{-} e G F P$ specific primer with $5^{\prime}$ end extension coding for the p2A peptide and a SmaI restriction site just downstream of p2A for convenient subcloning (pME_eGFP_p2A_fw and pME_eGFP_p2A_rev primer).

pME entry clones used for gain-of-function experiments. $p M E \_e G F P$ p2A_SmaI was digested with SmaI and XhoI. The inserts vegfaa 165 , vegfC and sflt 1 were amplified from zebrafish cDNA using primers vegfaa_p2A_fw/rev, vegfc_p2A_fw/rev and sflt1_p2A_fw/rev. The PCR products were digested with Xhol and gel purified. Vector and inserts were ligated following the manufactures instructions (NEB T4 DNA Ligase). The resulting plasmids were named $p M E \_e G F P$ p2A_vegfaa165, pME_eGFP-p2A_vegfc and $p M E \_e G F P-p 2 A \_s f l t 1$.

gal4ERt2 middle entry clone generation. To spatially and temporally regulate transgene expression an inducible gal4-ERT2 fusion protein was constructed. The Gal4 DNA binding domain was fused at its C-terminus with a mutant oestrogen ligand-binding domain ERT2 that carries a VP16-derived non-deleterious transactivation domain TA4 (ta4, 39 aa) at its C-terminus ${ }^{65}$. Among all possible sequential orders of domains, this arrangement was inferred to have a low background with a high induction rate ${ }^{66}$. A middle entry clone $p E N T R / D-c r e E R T 2$ was modified by replacing Cre recombinase domain $(1,053 \mathrm{bp}$, flanked by NotI and Xhol sites at its $5^{\prime}$ and $3^{\prime}$ termini, respectively) with a PCR product encoding Gal4 DNA binding domain (1-146 aa) with Kozak sequence in the $5^{\prime}$ vicinity of the start codon. To replace the stop codon at the $3^{\prime}$ terminus of ERT2 domain with TA4 domain, a C-terminal half of a ERT2 domain (115-316 aa, flanked by in-frame $\mathrm{NcoI}$ at its $5^{\prime}$ terminus) was replaced with a PCR product encoding the C-terminal half of the ERT2 domain without a stop codon (115-315 aa) with in-frame AgeI site at its $3^{\prime}$ terminus. The AgeI site, and $3^{\prime}$ downstream EcoRI site were utilized to insert two synthetic double-stranded oligonucleotides encoding the TA4 domain and the stop codon.

Generation of gateway expression clones. $p M E \_D N-M A M L-e G F P$ was kindly provided by Caroline Burns ${ }^{47}$. $p 5 E \_f l t 1^{\text {enh }}, p M E \_D N-M A M L-e G F P$ and p3E_polyA were recombined into pDestTol2CG2 according to the manufacturer's instructions (Thermo Fisher, LR Clonase II plus). The resulting plasmid was named pCG2_flt1_DN-MAML-eGFP. p5E_Xla.Tubb-3.8, pME_eGFP-p2A_sflt1 and p3E_polyA were recombined into pDestTol2CG2 (pCG2_Xla.Tubb-3.8_eGFP-p2A-sflt1).p5E_Xla.Tubb-3.8, pME_eGFP-p2A_sflt1 
and $p 3 E \_p o l y A$ were recombined into pDestTol2CG2 (pCG2_Xla.Tubb-3.8_eGFPp2A-sflt1).p5E_elavl-3.2 (R.W., unpublished observations), $p M E \_$gal4ERT2 and p3E_polyA were recombined into pDestTol2CG2 (pCG2_elavl-3.2_gal4-ERT2). $p 5 E_{-} f t 1^{\text {enh }}, p M E_{-}$gal4ERT2 and $p 3 E_{-} p o l y A$ were recombined into pDestTol2CG2 ( $p$ CG2 flt $^{\text {enh }}$ gal4-ERT2).

Generation of tissue-specific KO constructs. $p M E-C a s 9-T 2 A-G F P$ and pDestTol2pA2-U6:gRNA were a gift from Leonard Zon (Addgene plasmid \# 63156 and \# 63155 ${ }^{49}$. pDestTol2pA2-U6:gRNA ${ }^{f t 1 E 3}$ was generated by annealed oligo cloning. Oligos U6_flt1E3_1 and U6_flt1E3_2 were cloned into pDestTol2pA2-U6:gRNA following BseRI restriction digest. To drive Cas 9 expression specifically in neurons, the Gal4 driver construct $p C G 2$ Xla:Tubb3.8_gal4ERT2 was generated by recombining p5E_Xla.Tubb-3.8, pME_gal4ERT2, p3E_polyA and pDestTol2CG2. To drive Cas9 expression specifically in endothelial cells, the Gal4 driver construct $p C G 2 \_f l t 1^{\text {enh }}$ gal4ERT2 was generated by recombining $p 5 E_{-}$flt $1^{e n h}, p M E_{-}$gal4ERT2, $p 3 E_{-}$polyA and pDestTol2CG2. For the Gal4 effector construct, $p 5 E \_U A S, p M E$ cas9-t2a-eGFP and $p 3 E$ polyA were recombined into pDestTol2pA2-U6:sgRNA ${ }^{\text {flt1E3 }}$ ( $p$ CG2_UAS_Cas9-t2A$\left.e G F P \_U 6 \_g R N A^{f t 1 E 3}\right)$.

Tissue-specific miR155-fIt1-1-2-3 knockdown constructs. sflt $13^{\prime} \mathrm{UTR}$-specific miRNAs were designed using the BLOCK-IT RNAi Designer website (https://rnaidesigner.thermofisher.com/rnaiexpress/). To enhance miRNA effectiveness three sflt1 $3^{\prime}$ UTR-specific target sites with miRNA155 backbone were cloned in series. A fragment containing the three multiplexed miRNAs were synthesized by Eurofins Genomics and cloned into 641-pMER-GFP-miR155empty and 641-pMER-DsRED-miR155empty using restriction enzymes BamHI and $\mathrm{Xhol}^{50}$. The target sites are listed in Supplementary Table 7. The expression construct with Xla.Tubb or flt $1^{\text {enh }}$ promoter was cloned using gateway cloning. p5E_Xla.Tubb-3.8, 641-pMER-GFP-miR155-sflt1-1-2-3 and p3E_polyA were recombined into pDestTol2CG2 (pCG2_Xla.Tubb_GFP-miR155-sflt1-1-2-3). $p 5 E_{f} f t 1^{\text {enh }}, 641-p M E R-D s R e d-m i R 155-s f l t 1-1-2-3$ and $p 3 E \_p o l y A$ were recombined into pDestTol2CG2 ( $p$ CG2_flt1 ${ }^{\text {enh }} \_$DsRed-miR155-sflt1-1-2-3).

FACS. Approximately 500 embryos $T g(m n x 1: G F P)^{m l 2}, T g(H u C: E G F P)^{a s 8}$, $\mathrm{Tg}$ (Xla.Tubb:DsRed) ${ }^{z f 148}$ or vhl MO injected Tg(Xla.Tubb:DsRed) ${ }^{z f 148}$ embryos were dechorionated at $24 \mathrm{hpf}$ using pronase $(0.5 \mathrm{mg} / \mathrm{ml})$. Cells were dissociated using FACSMax as recommended by the manufacturer. $\mathrm{Tg}(m n x 1: G F P)^{m / 2}$, $\mathrm{Tg}(\mathrm{HuC}: E G F P)^{a s s}$ embryos were dissociated and sorted at $24 \mathrm{hpf}$, control and vhl MO injected $\mathrm{Tg}(\mathrm{Xla} \text {.Tubb:DsRed })^{z f 148}$ embryos were dissociated and sorted at 3 dpf. Dissociated cells were FACS sorted using BD-FACS-Aria I and Aria II. The sorted cells $\left(\sim 0.5 \times 10^{6}\right.$ cells per experiment $)$ were spun down at $310 \mathrm{~g}$ for $5 \mathrm{~min}$ and resuspended in lysis buffer contained in the RNeasy mini kit (Qiagen). RNA was extracted as described in the manual. Because of limited amounts of RNA the QuantiTect Whole Transcriptome Kit (Qiagen) was used to preamplify and reverse transcribe the RNA to make CDNA. CDNA was diluted 1:250 for real-time $\mathrm{qPCR}$

\section{Gene expression analysis by real-time qPCR and TaqMan. Total RNA of} zebrafish embryos was isolated with TRIzol, purified with RNeasy mini kit (Qiagen) and quantity and quality were measured using an Agilent 2,100 Bioanalyzer (Agilent Technologies) according to the manufacturer's instructions. We performed DNase on-column digestion using RNase-free DNase Set (Qiagen) according to the manufacturer, followed by cDNA synthesis using the Thermoscript First-Strand Synthesis System (Thermo Fisher Scientific). Primer probe sets (FAM and TAMRA labels) were obtained from Thermo Fisher Scientific. Amplification was carried out using an ABI Prism 7,000 thermocycler (Applied Biosystems). qPCR was conducted with SYBR Green PCR Master Mix (Thermo Scientific) in a StepOnePlus real-time qPCR system (Applied Biosystems). Primers for real-time qPCR were ordered from Eurofins Genomics. Gene expression data were normalized against zebrafish elongation factor 1-alpha. Primers and probes are listed in Supplementary Table 1-3.

RNA-seq library preparation and sequencing. Zebrafish RNA was isolated and purified from $4 \mathrm{dpf}$ zebrafish larvae using TRIzol and RNeasy mini kit (Qiagen) as recommended by the manufacturers. A cDNA library was generated using the TruSeq Ilumina RNA sample prepv2 kit according to the manufacturer's protocol. The cDNA library was sequenced on a HiSeq2000 according to the manufacturer's protocols (Illumina).

Identification of differentially expressed genes. Raw sequencing reads were mapped to the transcriptome and the zebrafish reference genome (GRCz10 danRer10) using Bowtie2.0 and TopHat 2.0 (ref. 67). On average 44,490,573 reads (81,6\% of total reads) were assigned to genes with Cufflinks and HTSeq software package. Differentially expressed genes (control vs. mutant) were identified using DESeq and Cuffdiff ${ }^{67,68}$. Genes were defined as differentially expressed if $\geq 2$ fold significantly regulated $(P<0.05)$ with two independent methods (DEseq and Cuffdiff).

Zebrafish histological sectioning. Dechorionated larvae were fixed in $4 \% \mathrm{PFA}$ for $2 \mathrm{~h}$ and subsequently transferred to $20 \% \mathrm{DMSO} / 80 \%$ Methanol and incubated overnight at $-20^{\circ} \mathrm{C}$. Larvae were then washed in $100 \mathrm{mM} \mathrm{NaCl}, 100 \mathrm{mMTris}-\mathrm{HCl}$, $\mathrm{pH} 7.4$ for $30 \mathrm{~min}$ at room temperature. Washed larvae were embedded in gelatin from cold water fish skin/sucrose (Sigma). Larvae were sectioned $(20 \mu \mathrm{m})$ in a cryomicrotome.

Inhibitor treatments. All stock solutions were prepared in DMSO. Embryos were dechorionated at $24 \mathrm{hpf}$ using Pronase (Roche, Basel, Switzerland). For Notch signalling inhibition embryos were incubated from $2 \mathrm{dpf}$ with $10 \mu \mathrm{M}$ of LY-411575 (Sigma, St Louis, MO, USA) and imaged at $3 \mathrm{dpf}$. For VEGFR2 and VEGFR3 inhibition embryos were treated with $25 \mu \mathrm{M}$ MAZ51 (Merck Millipore, Billerica, Massachusetts, USA) from $2.5 \mathrm{dpf}$ or from $3 \mathrm{dpf}$ with $0.125 \mu \mathrm{M}$ ki8751 (Sigma, St Louis, MO, USA) and imaged at $4 \mathrm{dpf}$. To inhibit PI3K/Akt signalling embryos were incubated with $1.25 \mu \mathrm{M}$ wortmannin from $3 \mathrm{dpf}$ and imaged at analysed at $4 \mathrm{dpf}$. Heartbeat was blocked using $15 \mathrm{mM}$ 2,3-Butanedione 2-monoxime (BDM) dissolved in E3 media. Control embryos were mock treated with DMSO (Sigma, St Louis, MO, USA). Embryos were randomly assigned to experimental groups. Investigators were blinded to inhibitor treatment.

Photoconversion of kikGR and migration tracking. Dechorionated embryos were embedded in $0.7 \%$ low-melting agarose at $30 \mathrm{hpf}$ and a small part of the posterior cardinal vein of $T g(k d r l: n l s k i k G R)^{h s c 7}$ transgenics was converted for several seconds using UV-light with the smallest available field diaphragm of the Leica Sp8 confocal microscope. Subsequently embryos removed from the agarose and allowed to develop in E3 medium until imaging or were immediately used for time-lapse imaging.

Gal4ERT2 endoxifen activation. Endoxifen (Sigma) was solved in DMSO. Zebrafish embryos expressing Gal4ERT2 were incubated from $52 \mathrm{hpf}$ onwards in $0.5 \mu \mathrm{M}$ endoxifen in E3 medium in the dark. GFP positive cells could be observed approximately $1.5 \mathrm{~h}$ after induction.

Vascular network analysis. To assess sprout number and length, we developed a semi-automated analysis of the DLAV-ISV vessel network using ImageJ (Supplementary Fig. 1n). Image-stacks of ISVs were acquired using the Leica SP8 confocal microscope. Stack projections of one side of the trunk were generated. Dorsal region of the ISVs was used for analysis. Using ImageJ a Gaussian blur filter was applied followed by a black/white threshold and subsequent skeletonization to generate a skeleton of the vasculature. Segment number, branch point number and total branch length were calculated using the 'analyse skeleton' plugin. The semi-automated pipeline was applied for analysis of $4 \mathrm{dpf}$ vascular networks, while sprout numbers in 2-3 dpf zebrafish embryos were counted manually.

Imaging. Zebrafish larvae were embedded in $0.7 \%$ low-melting agarose with $0.112 \mathrm{mg} \mathrm{ml}^{-1}$ Tricaine (E10521, Sigma) and $0.003 \%$ PTU (P7629, Sigma) in glass bottom dishes (MatTek, P35G-0.170-14-C). Images presented in this study were acquired using a Leica SP8 confocal microscope with $\times 20$ multi-immersion and $\times 40$ water immersion objectives and LAS X software. Images were processed using ImageJ. Vascular branching was quantified using a semi-automated ImageJ pipeline (Supplementary Fig. 1n). Animal numbers used are indicated in figure legends. For zebrafish mutants more than 100 embryos per genotype were analysed. In morpholino experiments morphologically malformed embryos were excluded from analysis.

Statistical analysis. Statistical analysis was performed using GraphPad Prism 6. Each dataset was tested for normal distribution (D'Agostino and Pearson test). Parametric method (unpaired Students $t$-test) was only applied if the data were normally distributed. For non-normal distributed data sets, a non-parametric test (Mann Whitney $U$ test) was applied. When appropriate in case of multiple comparisons, ANOVA plus Bonferroni correction was applied. $P$ values $<0.05$ were considered significant. Data are represented as mean \pm s.e.m., unless otherwise indicated. ${ }^{*} P<0.05,{ }^{* *} P<0.01$ and ${ }^{* * *} P<0.001$.

Data availability. The authors declare that all data supporting the findings of this study are available within the article and its Supplementary Information files or from the corresponding author on reasonable request. The RNA-seq data generated in this study has been deposited into the Gene Expression Omnibus database with the accession code http://www.ncbi.nlm.nih.gov/geo/query/ acc.cgi?acc $=$ GSE 89350 


\section{References}

1. Zacchigna, S., Lambrechts, D. \& Carmeliet, P. Neurovascular signalling defects in neurodegeneration. Nat. Rev. Neurosci. 9, 169-181 (2008).

2. White, I. A., Gordon, J., Balkan, W. \& Hare, J. M. Sympathetic reinnervation is required for mammalian cardiac regeneration. Circ. Res. 117, 990-994 (2015).

3. Nam, J. et al. Coronary veins determine the pattern of sympathetic innervation in the developing heart. Development 140, 1475-1485 (2013).

4. Eichmann, A., Le Noble, F., Autiero, M. \& Carmeliet, P. Guidance of vascular and neural network formation. Curr. Opin. Neurobiol. 15, 108-115 (2005).

5. Larrivee, B., Freitas, C., Suchting, S., Brunet, I. \& Eichmann, A. Guidance of vascular development: Lessons from the nervous system. Circ. Res. 104, 428-441 (2009).

6. James, J. M. \& Mukouyama, Y. Neuronal action on the developing blood vessel pattern. Semin. Cell Dev. Biol. 22, 1019-1027 (2011).

7. Schratzberger, P. et al. Reversal of experimental diabetic neuropathy by VEGF gene transfer. J. Clin. Invest. 107, 1083-1092 (2001)

8. Hogan, K. A., Ambler, C. A., Chapman, D. L. \& Bautch, V. L. The neural tube patterns vessels developmentally using the VEGF signaling pathway. Development 131, 1503-1513 (2004).

9. James, J. M., Gewolb, C. \& Bautch, V. L. Neurovascular development uses VEGF-A signaling to regulate blood vessel ingression into the neural tube. Development 136, 833-841 (2009).

10. Raab, S. et al. Impaired brain angiogenesis and neuronal apoptosis induced by conditional homozygous inactivation of vascular endothelial growth factor. Thromb. Haemost. 91, 595-605 (2004)

11. Mukouyama, Y. S., Shin, D., Britsch, S., Taniguchi, M. \& Anderson, D. J. Sensory nerves determine the pattern of arterial differentiation and blood vessel branching in the skin. Cell 109, 693-705 (2002).

12. Mukouyama, Y.-S., Gerber, H.-P., Ferrara, N., Gu, C. \& Anderson, D. J. Peripheral nerve-derived VEGF promotes arterial differentiation via neuropilin 1-mediated positive feedback. Development 132, 941-952 (2005).

13. Carmeliet, P. et al. Abnormal blood vessel development and lethality in embryos lacking a single VEGF allele. Nature 380, 435-439 (1996).

14. Miquerol, L., Langille, B. L. \& Nagy, A. Embryonic development is disrupted by modest increases in vascular endothelial growth factor gene expression. Development 127, 3941-3946 (2000).

15. Okabe, K. et al. Neurons limit angiogenesis by titrating VEGF in retina. Cell 159, 584-596 (2014).

16. Olsson, A.-K., Dimberg, A., Kreuger, J. \& Claesson-Welsh, L. VEGF receptor signalling in control of vascular function. Nat. Rev. Mol. Cell Biol. 7, 359-371 (2006).

17. Shibuya, M. Vascular endothelial growth factor (VEGF) and its receptor (VEGFR) signaling in angiogenesis: a crucial target for anti- and pro-angiogenic therapies. Genes Cancer 2, 1097-1105 (2011).

18. Krueger, J. et al. Flt1 acts as a negative regulator of tip cell formation and branching morphogenesis in the zebrafish embryo. Development 138, 2111-2120 (2011).

19. Jakobsson, L. et al. Endothelial cells dynamically compete for the tip cell position during angiogenic sprouting. Nat. Cell Biol. 12, 943-953 (2010).

20. Isogai, S., Lawson, N. D., Torrealday, S., Horiguchi, M. \& Weinstein, B. M. Angiogenic network formation in the developing vertebrate trunk. Development 130, 5281-5290 (2003).

21. Jin, S.-W., Beis, D., Mitchell, T., Chen, J.-N. \& Stainier, D. Y. R. Cellular and molecular analyses of vascular tube and lumen formation in zebrafish. Development 132, 5199-5209 (2005).

22. Covassin, L. D., Villefranc, J. A., Kacergis, M. C., Weinstein, B. M. \& Lawson, N. D. Distinct genetic interactions between multiple Vegf receptors are required for development of different blood vessel types in zebrafish. Proc. Natl Acad. Sci. USA 103, 6554-6559 (2006).

23. Liang, D. et al. The role of vascular endothelial growth factor (VEGF) in vasculogenesis, angiogenesis, and hematopoiesis in zebrafish development. Mech. Dev. 108, 29-43 (2001).

24. Covassin, L. D. et al. A genetic screen for vascular mutants in zebrafish reveals dynamic roles for Vegf/Plcg1 signaling during artery development. Dev. Biol. 329, 212-226 (2009).

25. Siekmann, A. F. \& Lawson, N. D. Notch signalling limits angiogenic cell behaviour in developing zebrafish arteries. Nature 445, 781-784 (2007).

26. Hogan, B. M. et al. Vegfc/Flt4 signalling is suppressed by Dll4 in developing zebrafish intersegmental arteries. Development 136, 4001-4009 (2009)

27. Villefranc, J. A. et al. A truncation allele in vascular endothelial growth factor c reveals distinct modes of signaling during lymphatic and vascular development. Development 140, 1497-1506 (2013)

28. Hiratsuka, S., Minowa, O., Kuno, J., Noda, T. \& Shibuya, M. Flt-1 lacking the tyrosine kinase domain is sufficient for normal development and angiogenesis in mice. Proc. Natl Acad. Sci. USA 95, 9349-9354 (1998).

29. Hiratsuka, S. et al. Membrane fixation of vascular endothelial growth factor receptor 1 ligand-binding domain is important for vasculogenesis and angiogenesis in mice. Mol. Cell. Biol. 25, 346-354 (2005).
30. Avraham-Davidi, I. et al. ApoB-containing lipoproteins regulate angiogenesis by modulating expression of VEGF receptor 1. Nat. Med. 18, 967-973 (2012).

31. Rossi, A. et al. Genetic compensation induced by deleterious mutations but not gene knockdowns. Nature 524, 230-233 (2015).

32. Chappell, J. C., Taylor, S. M., Ferrara, N. \& Bautch, V. L. Local guidance of emerging vessel sprouts requires soluble Flt-1. Dev. Cell 17, 377-386 (2009).

33. Gundersen, G. G. \& Worman, H. J. Nuclear positioning. Cell 152, 1376-1389 (2013).

34. Herbert, S. P. \& Stainier, D. Y. R. Molecular control of endothelial cell behaviour during blood vessel morphogenesis. Nat. Rev. Mol. Cell Biol. 12, 551-564 (2011).

35. Quillien, A. et al. Distinct Notch signaling outputs pattern the developing arterial system. Development 141, 1544-1552 (2014).

36. del Toro, R. et al. Identification and functional analysis of endothelial tip cellenriched genes. Blood 116, 4025-4033 (2010).

37. Strasser, G. A., Kaminker, J. S. \& Tessier-Lavigne, M. Microarray analysis of retinal endothelial tip cells identifies CXCR4 as a mediator of tip cell morphology and branching. Blood 115, 5102-5110 (2010).

38. Okuda, K. S. et al. lyve1 expression reveals novel lymphatic vessels and new mechanisms for lymphatic vessel development in zebrafish. Development 139, 2381-2391 (2012).

39. Bussmann, J. et al. Arteries provide essential guidance cues for lymphatic endothelial cells in the zebrafish trunk. Development 137, 2653-2657 (2010).

40. Nicenboim, J. et al. Lymphatic vessels arise from specialized angioblasts within a venous niche. Nature 522, 56-61 (2015).

41. Carmeliet, P. et al. Role of HIF-1alpha in hypoxia-mediated apoptosis, cell proliferation and tumour angiogenesis. Nature 394, 485-490 (1998).

42. van Rooijen, E. et al. von Hippel-Lindau tumor suppressor mutants faithfully model pathological hypoxia-driven angiogenesis and vascular retinopathies in zebrafish. Dis. Model. Mech. 3, 343-353 (2010).

43. Gomez-Manzano, C. et al. Mechanisms underlying PTEN regulation of vascular endothelial growth factor and angiogenesis. Ann. Neurol. 53, 109-117 (2003).

44. Choorapoikayil, S., Weijts, B., Kers, R., de Bruin, A. \& den Hertog, J. Loss of Pten promotes angiogenesis and enhanced vegfaa expression in zebrafish. Dis. Model. Mech. 6, 1159-1166 (2013).

45. Leslie, J. D. et al. Endothelial signalling by the Notch ligand Delta-like 4 restricts angiogenesis. Development 134, 839-844 (2007).

46. Suchting, S. et al. The Notch ligand Delta-like 4 negatively regulates endothelial tip cell formation and vessel branching. Proc. Natl Acad. Sci. USA 104, 3225-3230 (2007).

47. Zhao, L. et al. Notch signaling regulates cardiomyocyte proliferation during zebrafish heart regeneration. Proc. Natl Acad. Sci. USA 111, 1403-1408 (2014)

48. Stahlhut, C., Suárez, Y., Lu, J., Mishima, Y. \& Giraldez, A. J. miR-1 and miR 206 regulate angiogenesis by modulating VegfA expression in zebrafish. Development 139, 4356-4364 (2012)

49. Ablain, J., Durand, E. M., Yang, S., Zhou, Y. \& Zon, L. I. A CRISPR/Cas9 vector system for tissue-specific gene disruption in zebrafish. Dev. Cell 32, 756-764 (2015).

50. Giacomotto, J., Rinkwitz, S. \& Becker, T. S. Effective heritable gene knockdown in zebrafish using synthetic microRNAs. Nat. Commun. 6, 7378 (2015).

51. Childs, S., Chen, J.-N., Garrity, D. M. \& Fishman, M. C. Patterning of angiogenesis in the zebrafish embryo. Development 129, 973-982 (2002).

52. Kwon, H.-B. H.-B. et al. The parallel growth of motoneuron axons with the dorsal aorta depends on Vegfc/Vegfr3 signaling in zebrafish. Development 140, 4081-4090 (2013).

53. Lange, C. et al. Relief of hypoxia by angiogenesis promotes neural stem cell differentiation by targeting glycolysis. EMBO J. 35, 924-941 (2016).

54. Wiley, D. M. et al. Distinct signalling pathways regulate sprouting angiogenesis from the dorsal aorta and the axial vein. Nat. Cell Biol. 13, 686-692 (2011).

55 . Hen, G. et al. Venous-derived angioblasts generate organ-specific vessels during zebrafish embryonic development. Development 142, 4266-4278 (2015).

56. Xu, C. et al. Arteries are formed by vein-derived endothelial tip cells. Nat. Commun. 5, 5758 (2014).

57. Red-Horse, K., Ueno, H., Weissman, I. L. \& Krasnow, M. A. Coronary arteries form by developmental reprogramming of venous cells. Nature 464, 549-553 (2010).

58. Hogan, B. et al. ccbe1 is required for embryonic lymphangiogenesis and venous sprouting. Nat. Genet. 41, 396-398 (2009).

59. Peri, F. \& Nüsslein-Volhard, C. Live imaging of neuronal degradation by microglia reveals a role for v0-ATPase al in phagosomal fusion in vivo. Cell 133, 916-927 (2008)

60. van Impel, A. et al. Divergence of zebrafish and mouse lymphatic cell fate specification pathways. Development 141, 1228-1238 (2014).

61. Ando, K. et al. Clarification of mural cell coverage of vascular endothelial cells by live imaging of zebrafish. Development 143, 1328-1339 (2016). 
62. Santhakumar, K. et al. A zebrafish model to study and therapeutically manipulate hypoxia signaling in tumorigenesis. Cancer Res. 72, 4017-4027 (2012).

63. Hwang, W. Y. et al. Efficient genome editing in zebrafish using a CRISPR-Cas system. Nat. Biotechnol. 31, 227-229 (2013).

64. Kwan, K. M. et al. The Tol2kit: a multisite gateway-based construction kit for Tol2 transposon transgenesis constructs. Dev. Dyn. 236, 3088-3099 (2007).

65. Distel, M., Wullimann, M. F. \& Köster, R. W. Optimized Gal4 genetics for permanent gene expression mapping in zebrafish. Proc. Natl Acad. Sci. USA 106, 13365-13370 (2009).

66. Louvion, J. F., Havaux-Copf, B. \& Picard, D. Fusion of GAL4-VP16 to a steroid-binding domain provides a tool for gratuitous induction of galactoseresponsive genes in yeast. Gene 131, 129-134 (1993).

67. Trapnell, C. et al. Differential gene and transcript expression analysis of RNAseq experiments with TopHat and Cufflinks. Nat. Protoc. 7, 562-578 (2012).

68. Anders, S. \& Huber, W. Differential expression analysis for sequence count data. Genome Biol. 11, R106 (2010).

\section{Acknowledgements}

We thank the colleagues of the KIT-European Zebrafish Resource Center (EZRC) for handling and maintenance of the zebrafish lines. We are very grateful to Dr Caroline Burns, Cardiovascular Research Center, Charlestown for sharing the pME_DN-MAMLGFP entry clone. We thank Dr Thomas Becker and Dr Jean Giacomotto, Brain and Mind Research Institute, University of Sydney for the 641-pMER-GFP/DsRed-miR155empty plasmids. We thank Leonard I. Zon M.D., Boston Children's Hospital and Dana Farber Cancer Institute, Boston for the pDestTol2CG2-U6:gRNA and the pME-Cas9-T2A-GFP constructs and Dr Keith Joung, Massachusetts General Hospital, Charlestown for DR274 and MLM3613 plasmids. We are also very grateful to Dr Jeroen den Hertog, Hubrecht Institute, Utrecht for sharing the zebrafish ptena $^{-/-} ;$pten $^{-/-}$double mutants. S.S.-M, U.S. and F.L.N. are members of the EuFishBioMed zebrafish initiative. S.S.-M. and F.I.N. are supported by grants from the Deutsche Forschungsgemeinschaft (DFG)-FOR2325 'Interactions at the Neurovascular Interface'. Y.H. is supported by an individual grant from the Danish Council for Independent Research. We acknowledge support by Deutsche Forschungsgemeinschaft and Open Access Publishing Fund of Karlsruhe Institute of Technology.

\section{Author contributions}

R.W. designed and performed experiments and interpreted experimental data. A.K. and R.W. performed and analysed FACS experiments. K.A. participated in manuscript preparation. L.P. performed and analysed inhibitor experiments. J.K. performed and analysed Taqman experiments. M.T. \& U.S. contributed the Gal4ERT2 construct, Y.H. performed FACS analyses, K.A. \& N.M. performed the analysis on pericyte contribution. A.van I. and S.S.-M. contributed transgenic fish, constructs, interpreted data and discussed the conceptual framework. F.I.N. conceived and designed the project, analysed the data and supervised the overall project. F.I.N. and R.W. wrote the manuscript.

\section{Additional information}

Supplementary Information accompanies this paper at http://www.nature.com/ naturecommunications

Competing financial interests: The authors declare no competing financial interests.

Reprints and permission information is available online at http://npg.nature.com/ reprintsandpermissions/

How to cite this article: Wild, R. et al. Neuronal sFlt1 and Vegfaa determine venous sprouting and spinal cord vascularization. Nat. Commun. 8, 13991 doi: 10.1038/ncomms13991 (2017).

Publisher's note: Springer Nature remains neutral with regard to jurisdictional claims in published maps and institutional affiliations.

(c) (i) This work is licensed under a Creative Commons Attribution 4.0 International License. The images or other third party material in this article are included in the article's Creative Commons license, unless indicated otherwise in the credit line; if the material is not included under the Creative Commons license, users will need to obtain permission from the license holder to reproduce the material. To view a copy of this license, visit http://creativecommons.org/licenses/by/4.0/

(C) The Author(s) 2017 